\title{
D-OPTIMAL EXPERIMENTAL DESIGN COUPLED WITH PARALLEL FACTOR ANAL YSIS 2 DECOMPOSITION A USEFUL TOOL IN THE DETERMINATION OF TRIAZINES IN ORANGES BY PROGRAMMED TEMPERATURE VAPORISATION-GC/MS WHEN USING DISPERSIVE-SOLID PHASE EXTRACTION
}

\author{
A. Herrero ${ }^{1}$, M.C. Ortiz ${ }^{1, *}$, L.A. Sarabia ${ }^{2}$ \\ ${ }^{1}$ Department of Chemistry, University of Burgos, Faculty of Sciences, \\ Pza. Misael Bañuelos s/n, 09001 Burgos, Spain \\ 2 Department of Mathematics and Computation, University of Burgos, Faculty of Sciences, \\ Pza. Misael Bañuelos s/n, 09001 Burgos, Spain
}

\begin{abstract}
The determination of triazines in oranges using a GC/MS system coupled to a programmed temperature vaporizer (PTV) inlet in the context of legislation is performed. Both pretreatment (using a Quick Easy Cheap Effective Rugged and Safe (QuEChERS) procedure) and injection steps are optimized using D-optimal experimental designs for reducing the experimental effort. The relative dirty extracts obtained and the elution time shifts make it necessary to use a PARAFAC2 decomposition to solve these two usual problems in the chromatographic determinations. The "second-order advantage" of the PARAFAC2 decomposition allows unequivocal identification according to document SANCO/12495/2011 (taking into account the tolerances for relative retention time and the relative abundance for the diagnostic ions), avoiding false negatives even in the presence of unknown co-eluents. The detection limits (CC $\alpha$ ) found, from 0.51 to $1.05 \mu \mathrm{g} \mathrm{kg}$, are far below the maximum residue levels (MRLs) established by the European Union for simazine, atrazine, terbuthylazine, ametryn, simetryn, prometryn and terbutryn in oranges. No MRL violations were found in the commercial oranges analysed.
\end{abstract}

Keywords: PARAFAC2, experimental design, QuEChERS, PTV-GC/MS, triazines in oranges, SANCO/12495/2011.

\section{Introduction}

The setting of low harmonized maximum residue limits (MRLs) for pesticides in food [1] and the need of controlling their residues in a large number of food samples have highlighted the problem of working with complex matrices which require pretreatment stages to eliminate interferent compounds. Procedures including partitioning with organic solvents, adsorption chromatography and gel permeation chromatography or solid-phase extraction have been developed. However, the introduction of these steps causes an increase in the time of analysis, high consumption of organic non-environmentally friendly solvents and a source of losses in the analytical recoveries [2].

Different techniques have been developed to address this problem, among them the approach known as the Quick Easy Cheap Effective Rugged and Safe (QuEChERS) multiresidue methodology first reported in 2003 [3]. Compared to traditional approaches, this approach is a

\footnotetext{
*Corresponding author. e-mail: mcortiz@ubu.es. Tel.: 34 947259571. Fax: 34947258831.
} 
rapid, straightforward, and cost-effective sample preparation procedure with which a large number of samples can be processed simultaneously.

The QuEChERS approach typically involves an extraction with acetonitrile followed by a clean-up step (which is not always necessary [3]) which consists of a dispersive solid-phase extraction (dSPE); the final determination is carried out by gas or liquid chromatography (GC or LC) coupled to mass spectrometry (MS), taking the advantage of the high selectivity and sensitivity provided by these techniques. Although the QuEChERS method was initially developed for determining multiclass pesticides in fruits and vegetables [4], currently modifications of the original method [5,6,7] and applications for a wide variety of analytes in a wide variety of matrices can be found in the literature. For example, it has been applied for the extraction of veterinary drugs in fish [8], beef muscle [9], milk and liver [10], for the extraction of chlorinated compounds from soil samples [11] and of drugs in blood [12].

But the use of this multiresidue method increases the presence of co-extracts. Even after dSPE clean-up, QuEChERS extracts are relatively dirty because of the risk of removing pesticides along with other matrix compounds if refined clean-up steps are used, so the extracts can still contain co-extracted compounds which could interfere with the detection and identification of target analytes. The document SANCO/12495/2011 [13] recommends using "the ion that shows the best signal-to-noise ratio and no evidence of significant chromatographic interference" to quantify residues, specifying that an ion chromatogram that "shows evidence of significant chromatographic interference must not be relied upon to quantify or identify residues". But the presence of non-target compounds can cause false negatives during pesticides identification [14], since the maximum permitted tolerances for relative ion abundances established in the regulations [13] for diagnostic ions will not be fulfilled if some fragments of the non-target compounds contribute inadvertently to the abundance of some of the $\mathrm{m} / \mathrm{z}$ ratios of the pesticides.

The problem of overlapping peaks in GC/MS or LC/MS can be approached using parallel factor analysis (PARAFAC), which makes discrimination possible from co-eluting matrix components if the data are trilinear [14]. The PARAFAC decomposition provides the same number of factors as there are compounds whose signal is higher than the expected signal-tonoise ratio, as well as the mass spectrum and chromatographic profile of each compound. This includes compounds that co-elute and several artifacts like baseline. This is the "second-order advantage" of PARAFAC in chemical analysis. However, the PARAFAC model is greatly affected by deviations from the trilinear structure of the data, in such a way that slight changes in the retention time of an analyte between runs, which are usual in chromatography, lead to the invalidation of the PARAFAC model. For that reason, if some deviations in the chromatographic profiles have to be modelled, then the parallel factor analysis 2 (PARAFAC2) model, which was proposed in order to overcome this difficulty, must be used $[15,16]$. PARAFAC2 has the "second-order advantage" if the correlation between the retention times is the same in all samples. Applications of PARAFAC and PARAFAC2 to chromatographic analysis can be found in refs. [17,18,19].

In this work, we describe the determination of triazines in oranges. Orange samples were pretreated with a commercial kit for use with the QuEChERS method and next the extracts were analysed using a GC/MS system equipped with a programmed temperature vaporizer (PTV) inlet. Coupling this with large volume injections (LVI) the procedure can be improved since LVI techniques are a reliable alternative to carry out the preconcentration of samples inside the chromatographic system. For introducing this large volume of sample, repetitive or speed controlled injection can be used, being the latter the one that leads to better results [20]. 
For the optimization of the analytical procedure, traditional one-factor-at-a-time experiments do not address interactions among experimental factors; therefore experimental design strategies can be very helpful since a large number of factors are usually involved. Among them, D-optimal designs [21] make it easy the study of many experimental factors with a small number of experiments, and in addition they are a general methodology for making 'ad hoc' designs by adapting the experimental design to each analytical problem (it is possible to consider independently for each factor as many levels as it is required and their interactions) $[22,23,24]$.

Most of the works found in the literature dealing with the QuEChERS method optimization focused on the study of experimental conditions and/or the composition of salts, sorbents, etc. employed in the extraction/partitioning and/or clean-up steps [9,25]. However, if a commercial kit which contains tubes with pre-weighed sorbents and buffers for use with the QuEChERS method for a certain application is employed, as it is in this work, most of these parameters are already fixed. In this case, it is possible to optimize the sample preparation protocol provided by the supplier with the kit for that application, since it serves just as a guideline for a large number of compounds with different chemical properties. In this work, several parameters of the QuEChERS procedure have been optimized using a D-optimal design coupled to PARAFAC2 decomposition for selecting the best experimental conditions of the pretreatment. In addition, several experimental parameters of the injection step performed with the PTV inlet have also been optimized using another D-optimal design coupled, in this case, to PARAFAC decomposition.

124

125

126

127

128

129

130

131

132

133

134

The analytical procedure used for the determination of these triazines was validated and orange samples purchased from different food stores were analysed. The EU establishes MRLs for simazine (SZ), atrazine (AZ), terbuthylazine (TZ), simetryn (ST), ametryn (AT), prometryn (PT) and terbutryn (TT) in oranges [1,26,27], so compliance with those MRLs was checked. The MRLs $\left(0.10 \mathrm{mg} \mathrm{kg}^{-1}\right.$ for TZ, $0.05 \mathrm{mg} \mathrm{kg}^{-1}$ for AZ, and $0.01 \mathrm{mg} \mathrm{kg}^{-1}$ for SZ, ST, AT, PT and TT) are not exceeded in any case.

\section{Experimental}

\subsection{Chemicals and materials}

The triazines (SZ, AZ, PZ, TZ, ST, AT, PT, and TT; PESTANAL grade) were purchased from Sigma-Aldrich (Madrid, Spain). Methanol, ethyl acetate and acetonitrile (HPLC grade) were obtained from Merck (Darmstadt, Germany). All orange samples were purchased from local food stores.

QuEChERS method was performed using a DisQuE dispersive sample preparation kit from Waters (Milford, MA, USA), which consisted of $50 \mathrm{~mL}$ tubes containing $6 \mathrm{~g}$ anh. $\mathrm{MgSO}_{4}$ plus $1.5 \mathrm{~g}$ anh. sodium acetate (DisQuE extraction tube 1) and $2 \mathrm{~mL}$ tubes containing $150 \mathrm{mg}$ anh. $\mathrm{MgSO}_{4}$ plus $50 \mathrm{mg}$ PSA sorbent and $50 \mathrm{mg} \mathrm{C}_{18}$ for d-SPE clean-up (DisQuE clean-up tube 2).

\subsection{Instrumental}

The analyses were carried out on an Agilent (Agilent Technologies, Wilmington, DE, USA) 7890A gas chromatograph coupled to an Agilent 5975 Mass Selective Detector (MSD). The 
injection system consisted of a septumless head and a PTV inlet (CIS 6 from Gerstel, Mülheim an der Ruhr, Germany) which was equipped with an empty multi-baffled deactivated quartz liner. LVI was carried out using a MultiPurpose Sampler (MPS 2XL from Gerstel) with a $10 \mu \mathrm{L}$ syringe. Analytical separations were performed on an Agilent DB-5ms (30 $\mathrm{m} \times 0.25 \mathrm{~mm}$ i.d., $0.25 \mu \mathrm{m}$ film thickness) column. To centrifuge the extracts, a refrigerated tabletop centrifuge Sigma 2-16K (Osterode, Germany) was used. A miVac DUO centrifugal concentrator (Genevac Ltd., Ipswich, UK) operating at low pressure was used for faster evaporation.

\subsection{GC/MS conditions}

The PTV inlet was operated in the solvent vent mode. A volume of $10 \mu \mathrm{L}$ was injected at a controlled speed of $\mathrm{s} \mu \mathrm{L} \mathrm{s}^{-1}$ (injection speed). After each injection the syringe was washed several times first with acetone and next with ethyl acetate. During injection, the inlet temperature was held at $\mathrm{T} 1{ }^{\circ} \mathrm{C}$ (initial temperature) for $\mathrm{t} 1 \mathrm{~min}$ (PTV initial time), while the column head pressure was fixed to p psi (inlet $P$ ) and the flow rate through the split vent was set at $\mathrm{f} \mathrm{mL} \min ^{-1}$ (vent flow). At a solvent vent time of $\mathrm{t} 2 \mathrm{~min}$ (vent time) the split valve was closed. Next, the inlet temperature was ramped at $\mathrm{r}^{\circ} \mathrm{C} \mathrm{s}^{-1}$ (PTV rate) up to $\mathrm{T} 2{ }^{\circ} \mathrm{C}$ (end temperature), which was held for $3 \mathrm{~min}$. Afterwards, the temperature reached $280{ }^{\circ} \mathrm{C}$ at a rate of $1^{\circ} \mathrm{C} \mathrm{s}^{-1}$, and held at $280^{\circ} \mathrm{C}$ for $5 \mathrm{~min}$. The split valve was re-opened $2 \mathrm{~min}$ after injection to purge the inlet at a vent flow of $60 \mathrm{~mL} \mathrm{~min}^{-1}$. See Table 1 for the codification and levels of experimental variables or factors.

The oven temperature program was as follows: $40{ }^{\circ} \mathrm{C}$ (for $2 \mathrm{~min}$ ), temperature increase at 60 ${ }^{\circ} \mathrm{C} \min ^{-1}$ to $130{ }^{\circ} \mathrm{C}(0 \mathrm{~min})$, then $7{ }^{\circ} \mathrm{C} \mathrm{min}^{-1}$ to $160{ }^{\circ} \mathrm{C}(1 \mathrm{~min}), 3.5^{\circ} \mathrm{C} \mathrm{min} \mathrm{m}^{-1}$ to $178{ }^{\circ} \mathrm{C}(0$ min), and finally $50{ }^{\circ} \mathrm{C} \mathrm{min}{ }^{-1}$ to $220^{\circ} \mathrm{C}$ (2 min). A post-run step was performed for 4 min at $280^{\circ} \mathrm{C}$.

After 11 min (solvent delay), the mass spectrometer (MS) was operated in electron ionization (EI) mode at $70 \mathrm{eV}$ in selected ion monitoring (SIM) mode, with an acquisition window for each analyte. 5 ions (ion dwell time of $80 \mathrm{~ms}$ ) were detected for each peak: 158, 173, 186, 201 and 203 for SZ; 173, 200, 202, 215 and 217 for AZ; 172, 187, 214, 229 and 231 for PZ; 173, 214, 216, 229 and 231 for TZ; 155, 170, 198, 213 and 215 for ST; 170, 185, 212, 227 and 229 for AT; 184, 199, 226, 241 and 243 for PT; and 170, 185, 226, 241 and 243 for TT. The transfer line temperature was set at $250{ }^{\circ} \mathrm{C}$, the ion source temperature at $230{ }^{\circ} \mathrm{C}$, and the quadrupole at $150{ }^{\circ} \mathrm{C}$. The electron multiplier was set at $1576 \mathrm{~V}$ and the source vacuum at $10^{-5}$ Torr.

\subsection{Samples, standards and sample preparation procedure}

\subsubsection{Standards}

Stock solutions of the height triazines $\left(100 \mathrm{mg} \mathrm{L}^{-1}\right.$ of each triazine) were prepared in methanol and stored in a refrigerator at $4{ }^{\circ} \mathrm{C}$. Next, solutions of each triazine were prepared in ethyl acetate at a concentration of $1 \mathrm{mg} \mathrm{L}^{-1}$ from the stock solutions. Two sets of seven calibration standards were needed. Low level concentration calibration standards were prepared in ethyl acetate to each contain $10 \mu \mathrm{g} \mathrm{L}{ }^{-1}$ of the internal standard (PZ) plus $0,1,2,5$, 10, 15 and $20 \mu \mathrm{g} \mathrm{L}^{-1}$ of SZ, AZ, TZ, ST, AT, PT, and TT. High level concentration calibration standards were prepared in ethyl acetate to each contain $75 \mu \mathrm{g} \mathrm{L} \mathrm{L}^{-1}$ of the internal standard (PZ) plus $20 \mu \mathrm{g} \mathrm{L}^{-1}$ of AZ and $70 \mu \mathrm{g} \mathrm{L}^{-1}$ of TZ, or $30 \mu \mathrm{g} \mathrm{L}^{-1}$ of AZ and $80 \mu \mathrm{g} \mathrm{L}^{-1}$ of $\mathrm{TZ}$, or $40 \mu \mathrm{g} \mathrm{L}^{-1}$ of $\mathrm{AZ}$ and $90 \mu \mathrm{g} \mathrm{L} \mathrm{L}^{-1}$ of $\mathrm{TZ}$, or $50 \mu \mathrm{g} \mathrm{\textrm {L } ^ { - 1 }}$ of $\mathrm{AZ}$ and $100 \mu \mathrm{g} \mathrm{L}^{-1}$ of $\mathrm{TZ}$, or 60 
$\mu \mathrm{g} \mathrm{L}^{-1}$ of $\mathrm{AZ}$ and $110 \mu \mathrm{g} \mathrm{L}{ }^{-1}$ of $\mathrm{TZ}$, or $70 \mu \mathrm{g} \mathrm{L}^{-1}$ of $\mathrm{AZ}$ and $120 \mu \mathrm{g} \mathrm{L}^{-1}$ of $\mathrm{TZ}$, or $80 \mu \mathrm{g} \mathrm{L}^{-1}$ of $\mathrm{AZ}$ and $130 \mu \mathrm{g} \mathrm{L}^{-1}$ of $\mathrm{TZ}$ respectively.

\subsubsection{Samples}

The oranges were purchased from four different food stores (P1, P2, P3 and P4). Each orange was cut with a knife and put into a freezer overnight. The sample was blended while frozen until it reaches homogeneous texture. Then $15 \mathrm{~mL} \mathrm{5 \%}$ acetic acid (v/v) in acetonitrile and 15 $\mathrm{g}$ of the homogenized sample were added into the $50 \mathrm{~mL}$ DisQuE extraction tube 1 and after vortex mixing for $2 \mathrm{~min}$, the homogenate was centrifuged at $1500 \mathrm{rcf}, 10^{\circ} \mathrm{C}$ for $5 \mathrm{~min} .1 .2 \mathrm{~mL}$ of the acetonitrile extract was transferred into the DisQuE clean-up tube 2; the tube was shaken for $60 \mathrm{~s}$ and next centrifuged at $1500 \mathrm{rcf}, 10^{\circ} \mathrm{C}$ for $1 \mathrm{~min} .0 .5 \mathrm{~mL}$ of the supernatant were transferred into a tube and evaporated to dryness under vacuum in a centrifugal concentrator during $20 \mathrm{~min}$ at $50^{\circ} \mathrm{C}$. The residue was reconstituted with $500 \mu \mathrm{L}$ of ethyl acetate containing $10 \mu \mathrm{g} \mathrm{L}^{-1}$ of PZ as internal standard, filtered through Simplepure nylon membranes (13-mm diameter, 0.22- $\mu \mathrm{m}$, Membrane Solutions, Spring View Lane Plano, TX) and transferred into a vial with insert for analysis.

\subsubsection{Fortified samples}

Fortified orange samples were prepared following the procedure described in Section 2.4.2 for orange samples but in this case homogenized orange samples were fortified and vortex mixed for $30 \mathrm{~s}$ before extraction. A set of orange samples was fortified to each contain 0 or $10 \mu \mathrm{g} \mathrm{L}^{-1}$ of SZ, AZ, TZ, ST, AT, PT, and TT for low level concentration analysis, and another set was fortified with $0 \mu \mathrm{g} \mathrm{L}^{-1}$ of AZ and TZ, or $50 \mu \mathrm{g} \mathrm{L}{ }^{-1}$ of AZ plus $100 \mu \mathrm{g} \mathrm{L}{ }^{-1}$ of TZ for high level concentration analysis. The residue obtained though evaporation was reconstituted with 500 $\mu \mathrm{L}$ of ethyl acetate containing $10 \mu \mathrm{g} \mathrm{L}^{-1}$ of $\mathrm{PZ}$ as internal standard for low level concentration analysis and $75 \mu \mathrm{g} \mathrm{L}{ }^{-1}$ of PZ for high level concentration analysis

Another set of 13 fortified orange samples was prepared to optimize the QuEChERS procedure. These samples were fortified with $10 \mu \mathrm{g} \mathrm{L}^{-1}$ of SZ, AZ, TZ, ST, AT, PT, and TT and prepared following the procedure described previously but with some modifications according to the experimental design shown in Section 4.2 (see Table 2 for the codification and levels of experimental variables or factors). In this case, $15 \mathrm{~mL}$ of $\mathrm{m} \%$ of acetic acid $(\mathrm{v} / \mathrm{v})$ in acetonitrile (modifier) and $15 \mathrm{~g}$ of the homogenized sample were added into the tube 1 and after vortex mixing for $\mathrm{m} 1 \mathrm{~min}$ (mix_t1), the homogenate was centrifuged at $1500 \mathrm{rcf}$ and $10{ }^{\circ} \mathrm{C}$ for $\mathrm{m} 2$ min (centr_t1). $\mathrm{v} \mathrm{mL}$ of the acetonitrile extract (volume) was transferred into the tube 2 that was shaken for m3 s (mix_t2) and centrifuged at $1500 \mathrm{rcf}$ and $10{ }^{\circ} \mathrm{C}$ for 1 min. $0.5 \mathrm{~mL}$ of the supernatant were evaporated to dryness during $20 \mathrm{~min}$ at $\mathrm{e}^{\circ} \mathrm{C}$ (evap_T). The residue was reconstituted with $500 \mu \mathrm{L}$ of ethyl acetate containing $10 \mu \mathrm{g} \mathrm{L}^{-1}$ of PZ, filtered and transferred into a vial with insert for analysis.

\subsubsection{Matrix-matched standards}

10 matrix-matched standards were prepared following the procedure described in Section 
MSD ChemStation E.02.01.1177 (Agilent Technologies, Inc.) and Gerstel Maestro 1 (version 1.3.20.41/3.5) were used for data acquisition and processing. PARAFAC and PARAFAC2 models were performed with the PLS_Toolbox [28] for use with MATLAB version 7.10 (The MathWorks). The least squares regression models were built and validated with STATGRAPHICS Centurion XVI [29] and the least median of squares (LMS) regression models were fit with PROGRESS [30]. Decision limit, $\mathrm{CC} \alpha$, and capability of detection, CC $\beta$, were determined using the DETARCHI program [31], and CC $\alpha$ and CC $\beta$ at the maximum residue limit (MRL) were estimated using NWAYDET (a program written inhouse that evaluates the probabilities of false non-compliance and false compliance for n-way data). Both D-optimal experimental designs were built and analysed with NEMRODW [32]

\section{Theory}

\subsection{PARAFAC and PARAFAC2 models}

267

268

269

270

271

272

273

274

275

276

277

278

279

280

281

282

283

284

285

286

287

288

289

290

291

292

293

294

295

The PARAFAC decomposition is a method that decomposes a data tensor, $\underline{\mathbf{X}}$, into trilinear factors [33,34], each consisting of three loading vectors. GC/MS data can be arranged for each chromatographic peak in a three-way array or data tensor. In this case, the PARAFAC structural model for the abundance of each sample (slab k-th of $\underline{\mathbf{X}}$ ), is described in Eq. (1)

$X_{\mathrm{ijk}}=\sum_{f=1}^{\mathrm{F}} a_{\mathrm{if}} b_{\mathrm{jf}} c_{\mathrm{kf}}+e_{\mathrm{ijk}}, \quad \mathrm{i}=1, \ldots, \mathrm{I} ; \quad \mathrm{j}=1, \ldots, \mathrm{J} ; \quad \mathrm{k}=1, \ldots, \mathrm{K}$

where $\mathrm{F}$ is the number of factors (i.e. the total number of co-eluent analytes), $a_{\mathrm{if}}, b_{\mathrm{jf}}$ and $c_{\mathrm{kf}}$ are the elements of the three loadings matrices $\mathbf{A}, \mathbf{B}$ and $\mathbf{C}$, and $e_{\mathrm{ijk}}$ is the residual non explained by the trilinear model. In Eq. (1), $\boldsymbol{a}_{\mathrm{f}}$ is the chromatographic profile, $\boldsymbol{b}_{\mathbf{f}}$ is the spectral profile (the mass spectrum) and $\boldsymbol{c}_{\mathbf{f}}$ is the sample profile of the f-th analyte. Written in matrix notation the PARAFAC model, for the k-th slab of $\underline{\mathbf{X}}$, reads

$\mathbf{X}_{\mathrm{k}}=\mathbf{A D}_{\mathrm{k}} \mathbf{B}^{\mathrm{T}}+\mathbf{E}_{\mathrm{k}}, \mathrm{k}=1, \ldots, \mathrm{K}$

where $\mathbf{D}_{\mathrm{k}}$ is a diagonal matrix that holds the k-th row of matrix $\mathbf{C}$ in its diagonal, and $\mathbf{E}_{\mathrm{k}}$ is the matrix of the residuals.

The PARAFAC model assumes that the chromatographic and spectral profiles do not change shape in different experiments (only their magnitude) and the model fitted is highly affected if the structure of the data deviates considerably from this assumption. This trilinearity assumption can be relaxed in the chromatographic mode if the PARAFAC2 decomposition is used $[35,36]$. This is a slightly different decomposition method where the chromatographic profile depends also on the k-th sample, this solution being more accurate in this case. It is very rare to have alignment problems with MS data, but changes in retention times are very usual in chromatography [37]. The PARAFAC2 model can be expressed as follows:

$\mathbf{X}_{\mathrm{k}}=\mathbf{A}_{\mathrm{k}} \mathbf{D}_{\mathrm{k}} \mathbf{B}^{\mathrm{T}}+\mathbf{E}_{\mathrm{k}}=\mathbf{P}_{\mathrm{k}} \mathbf{H D}_{\mathrm{k}} \mathbf{B}^{\mathrm{T}}+\mathbf{E}_{\mathrm{k}}, \mathrm{k}=1, \ldots, \mathrm{K}$

where $\mathbf{D}_{\mathrm{k}}, \mathbf{B}^{\mathrm{T}}$, and $\mathbf{E}_{\mathrm{k}}$ are defined as in Eq. (2), $\mathbf{A}_{\mathrm{k}}(I \times F)$ are the chromatographic mode loadings estimated for the k-th sample, $\mathbf{P}_{\mathrm{k}}$ is an orthogonal matrix of the same size as $\mathbf{A}_{\mathrm{k}}$ and $\mathbf{H}$ is a small quadratic matrix with dimension equal to the number of components. In contrast 
to PARAFAC, PARAFAC2 does not assume that $\mathbf{A}$ is the same for all $\mathrm{k}$ but the cross-product matrix $\mathbf{A}_{k}{ }^{\mathrm{T}} \mathbf{A}_{k}$, which allows some deviation in the chromatographic profiles. PARAFAC2 has the "second-order advantage" if the correlation between the retention times is the same in all

\section{Results and discussion}

\subsection{Optimization of injection parameters}

It is well known that the introduction of the sample into the chromatographic system has a great influence on sensitivity, trueness and precision, especially when LVI techniques are used. For that reason in the first part of this work, the optimization of some injection parameters was carried out in order to obtain the highest chromatographic responses. The eight experimental variables detailed in Section 2.3 (injection speed, initial temperature, PTV initial time, inlet $P$, vent flow, vent time, PTV rate and end temperature), which may influence the PTV injection, were optimized. Table 1 shows the levels considered for the studied factors; there were 7 factors at two levels and one factor at 3 levels, such that 384 experiments would be necessary in a full design. Such a number of experiments was unaffordable; hence the experimental effort was reduced using a D-optimal design [22].

The aim of this experimental design procedure is to reduce the experimental effort just enough to estimate with suitable precision effects and interactions previously established. For that, once factors and their levels are established and a mathematical model is proposed (i.e. the search space is defined) an exchange algorithm based on the D-optimality criterion is used to look for experimental matrices with good quality [22,38]. In our case the search space was defined by the 384 experiments of the full factorial design. For each "n" (number of experiments to be done) the exchange algorithm searched among the 384 experiments the "n" experiments that led to the joint confidence region for the coefficients of the model with the smallest volume. The final number of experiments of the D-optimal design, "n", was chosen in such a way that the maximum of the variance inflation factors (VIFs) was close to 1 to guarantee the smallest possible variance for the estimated coefficients.

The mathematical reference-state model proposed for the response $y$ as a function of the studied factors was:

$\mathrm{y}=\beta_{0}+\beta_{1 \mathrm{~A}} \mathrm{x}_{1 \mathrm{~A}}+\beta_{2 \mathrm{~A}} \mathrm{x}_{2 \mathrm{~A}}+\beta_{3 \mathrm{~A}} \mathrm{x}_{3 \mathrm{~A}}+\beta_{4 \mathrm{~A}} \mathrm{x}_{4 \mathrm{~A}}+\beta_{5 \mathrm{~A}} \mathrm{x}_{5 \mathrm{~A}}+\beta_{6 \mathrm{~A}} \mathrm{x}_{6 \mathrm{~A}}+\beta_{7 \mathrm{~A}} \mathrm{x}_{7 \mathrm{~A}}+\beta_{8 \mathrm{~A}} \mathrm{x}_{8 \mathrm{~A}}+\beta_{8 \mathrm{~B}} \mathrm{x}_{8 \mathrm{~B}}+$ $\beta_{1 \mathrm{~A} 3 \mathrm{~A}} \mathrm{x}_{1 \mathrm{~A}} \mathrm{x}_{3 \mathrm{~A}}+\varepsilon$

where $\mathrm{x}_{\mathrm{ij}}(\mathrm{i}=1,2, \ldots, 8$ and $\mathrm{j}=\mathrm{A}, \mathrm{B})$ are binary variables equal to 1 when the $\mathrm{i}$-th factor is at the $\mathrm{j}$-th level, and 0 in any other case; $\beta_{0}$ is the intercept, $\beta_{\mathrm{ij}}$ are the coefficients of the model, and $\beta_{1 \mathrm{~A} \text { A }}$ is an additional coefficient to estimate the possible interaction between factors vent flow and vent time. The highest level was considered as the reference level for all the factors of the model (level C for the eighth factor and level B for the rest of factors); so the coefficients of this model measures the effect on the response when each factor changes from the reference level to another one. All the coefficients were estimated by least squares. 
The model of Eq. (4) had 11 coefficients, therefore at least 11 experiments were necessary to fit the model. In this case, the 384 possible experiments of the full factorial design were reduced to the 16 experiments (plus 3 replicates) of the D-optimal design shown in Table 3 (experiments 12, 13 and 14 are replicates of experiment 11), for which a first minimum of the maximum VIF was reached. The VIFs of the coefficients of the reduced model ranged between 1.00 and 1.24 , which meant that this design provides sufficiently precise estimates for the coefficients of the model.

Injections of the calibration standard of $10 \mu \mathrm{g} \mathrm{\textrm {L } ^ { - 1 }}$ of SZ, AZ, TZ, ST, AT, PT and TT, containing $10 \mu \mathrm{g} \mathrm{L}^{-1}$ of $\mathrm{PZ}$ as internal standard, were carried out according to the experimental plan (in random order) in Table 3. Fig. 1a shows the total ion chromatograms (TIC) acquired for experiments 2 and 11; absence of co-eluents and no shifting of the chromatographic peaks can be seen. Table 3 also contains the experimental responses, which are the standardized loadings of the sample mode calculated for each triazine through the PARAFAC decomposition of a data tensor obtained from the 19 experiments of the Doptimal design.

For obtaining these responses, GC/MS data from injections of the calibration standard according to the experimental design were arranged in a data tensor, and next PARAFAC decompositions were performed with them. For each experiment of the experimental plan, 5 $\mathrm{m} / \mathrm{z}$ ratios were acquired at a range of $\mathrm{J}$ times around the retention time of each triazine and the internal standard, in such a way that a data tensor with dimensions $I \times 5 \times 19$ was obtained for the chromatographic peak of each triazine after baseline correction. The first dimension of the datasets refers to the number of scans (I was 24, 21, 20, 18, 13, 10, 13 and 11 for SZ, AZ, $\mathrm{PZ}, \mathrm{TZ}, \mathrm{ST}, \mathrm{AT}, \mathrm{PT}$ and TT respectively), the second dimension to the number of $\mathrm{m} / \mathrm{z}$ ratios at which abundance was acquired, and the third dimension to the number of experiments. A PARAFAC model was built by applying the ALS algorithm to the tensor of each triazine and to the tensor of the internal standard; the non-negativity constraint was enforced for the three modes. Only one factor was necessary in all the PARAFAC decompositions, as expected since there were no interferences; the variance explained with the multi-way models ranged from 98.0 to $99.3 \%$.

For identification, the ratios of the loadings of the spectral profile of four diagnostic ions were calculated for each triazine (expressed as a percentage of the loading of each ion with respect to the highest loading, which corresponds to the base peak), and then the ratios were checked to see if they were within the tolerance intervals established for relative ion abundances according to the document SANCO/12495/2011 [13]. To calculate the permitted tolerance intervals (see Table 4) a standard which contained $10 \mu \mathrm{g} \mathrm{L}^{-1}$ of all the triazines was used as reference sample. For each triazine, there were at least three relative ion abundances (in fact four) within the tolerance intervals, as the regulation requires when working with a standard mass resolution detector in the SIM mode. Besides, the relative retention time (the ratio of the retention time of the chromatographic profile of each triazine to that the one of the internal standard) corresponded to that the one of the reference sample with a tolerance of $\pm 0.5 \%$ as document SANCO states. Since mass spectral and chromatographic profiles were unequivocally identified for the samples of the experimental plan. The use of the PARAFAC decomposition guaranteed a direct relation between the loadings of the sample profile and the amount of each triazine present in each sample. As usual in chromatography, the raw responses were standardized; the three way procedure for this task was developed and discussed in ref. [39]. The loadings of the sample profile were standardized by dividing each loading by the corresponding loading estimated for the internal standard (PZ) in the 
PARAFAC decomposition performed with the data tensor of PZ. These standardized loadings were the responses of the D-optimal design showed in Table 3.

The standardized loadings were used to fit the model of Eq. (4) for the seven triazines. Except for TZ, all the models were significant at $10 \%$ significance level (p-values $<0.10$; null hypothesis: the linear regression model is not significant) and did not have significant lack of fit at $5 \%$ significance level (p-values > 0.2; null hypothesis: the regression model adequately fits data). Data 12 was outlier in the models fit for PT and TT; therefore the final models for these triazines were fit without it (the VIFs of the coefficients of these models were between 1.05 and 1.33 after outliers elimination). In all cases, the residuals followed a normal distribution. The coefficients of determination ranged from 0.80 to 0.93 .

Coefficients of the reference-state model of Eq. (4) were estimated by least squares and depend on the reference state chosen. But for the analysis of effects, since the selection of the reference state is arbitrary, makes the interpretation of coefficients difficult because if the selected reference state changes, the estimated coefficients change too. This problem is avoided if a presence-absence model is used for that, so the reference-state model of Eq. (4) was converted into the equivalent presence-absence model of Eq (5).

$$
\begin{aligned}
& y=\beta_{0}^{\prime}+\beta_{1 \mathrm{~A}}^{\prime} \mathrm{x}_{1 \mathrm{~A}}+\beta_{1 \mathrm{~B}}^{\prime} \mathrm{x}_{1 \mathrm{~B}}+\beta_{2 \mathrm{~A}}^{\prime} \mathrm{x}_{2 \mathrm{~A}}+\beta_{2 \mathrm{~B}}^{\prime} \mathrm{x}_{2 \mathrm{~B}}+\beta_{3 \mathrm{~A}}^{\prime} \mathrm{x}_{3 \mathrm{~A}}+\beta_{3 \mathrm{~B}}^{\prime} \mathrm{x}_{3 \mathrm{~B}}+\beta_{4 \mathrm{~A}}^{\prime} \mathrm{x}_{4 \mathrm{~A}}+\beta_{4 \mathrm{~B}}^{\prime} \mathrm{x}_{4 \mathrm{~B}}+\beta_{5 \mathrm{~A}}^{\prime} \mathrm{x}_{5 \mathrm{~A}}+ \\
& \beta_{5 \mathrm{~B}}^{\prime} \mathrm{x}_{5 \mathrm{~B}}+\beta_{6 \mathrm{~A}}^{\prime} \mathrm{x}_{6 \mathrm{~A}}+\beta_{6 \mathrm{~B}}^{\prime} \mathrm{x}_{6 \mathrm{~B}}+\beta_{7 \mathrm{~A}}^{\prime} \mathrm{x}_{7 \mathrm{~A}}+\beta_{7 \mathrm{~B}}^{\prime} \mathrm{x}_{7 \mathrm{~B}}+\beta_{8 \mathrm{~A}}^{\prime} \mathrm{x}_{8 \mathrm{~A}}+\beta_{8 \mathrm{~B}}^{\prime} \mathrm{x}_{8 \mathrm{~B}}+\beta_{8 \mathrm{C}}^{\prime} \mathrm{x}_{8 \mathrm{C}}+\beta_{1 \mathrm{~A} 3 \mathrm{~A}}^{\prime} \mathrm{x}_{1 \mathrm{~A}} \mathrm{x}_{3 \mathrm{~A}}+
\end{aligned}
$$$$
\beta_{1 \mathrm{~A} 3 \mathrm{~B}}^{\prime} \mathrm{x}_{1 \mathrm{~A}} \mathrm{x}_{3 \mathrm{~B}}+\beta_{1 \mathrm{~B} 3 \mathrm{~A}}^{\prime} \mathrm{x}_{1 \mathrm{~B}} \mathrm{x}_{3 \mathrm{~A}}+\beta_{1 \mathrm{~B} 3 \mathrm{~B}}^{\prime} \mathrm{x}_{1 \mathrm{~B}} \mathrm{x}_{3 \mathrm{~B}}+\varepsilon
$$

The variables and coefficients in Eq. (5) have the same meaning as in Eq. (4) but now $\mathrm{j}=\mathrm{A}$, $\mathrm{B}, \mathrm{C}$; therefore it includes all the levels of the factors. In this case, each coefficient of Eq. (5) estimates the effect of the factor at the corresponding level on the response.

But the coefficients of the model of Eq. (5) cannot be estimated by least squares because for each factor ' $\mathrm{i}$ ' the sum $\mathrm{x}_{\mathrm{iA}}+\mathrm{x}_{\mathrm{iB}}$ (or $\mathrm{x}_{\mathrm{iA}}+\mathrm{x}_{\mathrm{iB}}+\mathrm{x}_{\mathrm{iC}}$, when there are three levels) equals 1 . However, once coefficients of Eq. (4) have been calculated, as a relationship exists between the coefficients of both presence-absence and reference state models, the coefficients of Eq. (5) can be computed. Ref. [22] contains more details about the equations that relate the coefficients of both models. Fig. 2 shows the graphic study of the effects of the different injection conditions on standardized loadings. The bars show through the differences of the coefficients, the expected change of the responses as effect of changes of the levels of each factor. The significant effects (at 5\% significance level) are those that are not within the interval depicted by the dash-dotted lines.

More significant effects were found for the most volatile triazines (SZ and AZ). The effect of the factor end temperature (coefficient $b_{7 A}$ ) was significant for the six triazines and had a negative sign for level $\mathrm{A}$ and a positive one for level B; i.e. the highest responses were achieved when injection was carried out at level B of this factor, namely this experimental parameter was set at $320^{\circ} \mathrm{C}$. The factor injection speed was also significant for three of the six models; in this case the maximum response was obtained for level $\mathrm{C}$ of the factor, i.e. when the sample was injected at $3.4 \mu \mathrm{L} \mathrm{s}^{-1}$. And also initial temperature and PTV rate were significant for some models; the lowest level of both factors led to the best responses, i.e. the optimum conditions for these two parameters were $40{ }^{\circ} \mathrm{C}$ and $10^{\circ} \mathrm{C} \mathrm{s}^{-1}$ respectively. The rest of the parameters under optimization as well as the interaction between vent flow and vent time had no significant influence on the considered response. However the working 
conditions were chosen bearing in mind the sign and size of the corresponding coefficients in Fig. 2 (taking into account the value of the majority) that maximized the response, so factors vent flow, inlet $P$, solvent vent time and PTV intial time were set to $100 \mathrm{~mL} \mathrm{~min}^{-1}$, 8 psi, 0.3 min, and $0.6 \mathrm{~min}$, respectively. The model fit for $\mathrm{TZ}$ was not significant because the variability of the standardized loadings of the replicates was of the same order as the one in the rest of experiments in the design, even if it was low. Therefore it was concluded that these experimental parameters do not interfere significantly the standardized loadings obtained for $\mathrm{TZ}$ in the experimental domain; the optimal conditions found for the rest of triazines can be applied also to the analysis of TZ.

\subsection{Optimization of the extraction parameters}

Once the injection parameters were optimized, the analysis of orange samples was tackled. Obviously, a pretreatment step was needed to put the samples into the chromatographic system; in this case the QuEChERS procedure was chosen. Taking as reference the procedure proposed by the supplier of the QuEChERS kit for the analysis of pesticides in oranges, six variables of the QuEChERS extraction procedure explained in Section 2.4.3 and Table 2 (mix_t1, centr_t1, volume, mix_t2, evap_T and modifier) were optimized.

The reference-state model fitted in this case was:

$\mathrm{y}=\beta_{0}+\beta_{1 \mathrm{~A}} \mathrm{x}_{1 \mathrm{~A}}+\beta_{2 \mathrm{~A}} \mathrm{x}_{2 \mathrm{~A}}+\beta_{3 \mathrm{~A}} \mathrm{x}_{3 \mathrm{~A}}+\beta_{4 \mathrm{~A}} \mathrm{x}_{4 \mathrm{~A}}+\beta_{5 \mathrm{~A}} \mathrm{x}_{5 \mathrm{~A}}+\beta_{6 \mathrm{~A}} \mathrm{x}_{6 \mathrm{~A}}+\beta_{6 \mathrm{~B}} \mathrm{x}_{6 \mathrm{~B}}+\varepsilon$

where $\mathrm{x}_{\mathrm{ij}}(\mathrm{i}=1,2, \ldots, 6$ and $\mathrm{j}=\mathrm{A}, \mathrm{B})$ were binary variables that equals 1 when the $i$ th factor is at the jth level, and 0 otherwise. $\beta_{0 \mathrm{i}} \mathrm{s}$ the intercept and $\beta_{\mathrm{ij}}$ are the coefficients of the model. The model also shows that the percentage of acetic acid in acetonitrile (modifier) was studied at three levels, and the remaining five factors were studied at two levels. The 96 possible experiments of the full factorial design were reduced to only 10 experiments (plus 3 replicates) using the D-optimal design methodology previously described. The corresponding design, shown in Table 5, had the VIFs of the coefficients with values between 1.09 and 1.11, so precise enough estimates for the coefficients of the model could be expected. Like in Section 4.1, the reference-state model of Eq. (6) was converted into the equivalent presenceabsence model of Eq (7).

$$
\begin{aligned}
& y=\beta_{0}^{\prime}+\beta_{1 \mathrm{~A}}^{\prime} \mathrm{x}_{1 \mathrm{~A}}+\beta_{1 \mathrm{~B}}^{\prime} \mathrm{x}_{1 \mathrm{~B}}+\beta_{2 \mathrm{~A}}^{\prime} \mathrm{x}_{2 \mathrm{~A}}+\beta_{2 \mathrm{~B}}^{\prime} \mathrm{x}_{2 \mathrm{~B}}+\beta_{3 \mathrm{~A}}^{\prime} \mathrm{x}_{3 \mathrm{~A}}+\beta_{3 \mathrm{~B}}^{\prime} \mathrm{x}_{3 \mathrm{~B}}+\beta_{4 \mathrm{~A}}^{\prime} \mathrm{x}_{4 \mathrm{~A}}+\beta_{4 \mathrm{~B}}^{\prime} \mathrm{x}_{4 \mathrm{~B}}+\beta_{5 \mathrm{~A}}^{\prime} \mathrm{x}_{5 \mathrm{~A}}+ \\
& \beta_{5 \mathrm{~B}}^{\prime} \mathrm{x}_{5 \mathrm{~B}}+\beta_{6 \mathrm{~A}}^{\prime} \mathrm{x}_{6 \mathrm{~A}}+\beta_{6 \mathrm{~B}}^{\prime} \mathrm{x}_{6 \mathrm{~B}}+\beta_{6 \mathrm{C}}^{\prime} \mathrm{x}_{6 \mathrm{C}}+\varepsilon
\end{aligned}
$$

Different pretreatments were carried out on the fortified orange samples (described in Section 2.4.3) setting the experimental conditions according to the experimental plan in Table 5. The obtained extracts were injected into the chromatographic system and the corresponding GC/MS signals were acquired (Fig. 1b shows the TIC acquired for experiment 4). There were shifts in the retention times and many co-eluents due to the relatively dirty extracts obtained with the QuEChERS procedure (a known disadvantage of using this pretreatment procedure). The high degree of both spectral and chromatographic interferences can cause false negatives during analyte identification, since the maximum permitted tolerances for relative ion abundances established in document SANCO/12495/2011 will not be fulfilled for at least 3 diagnostic ions. As an example, Fig. 3 shows, in dotted lines, the abundances acquired for the 5 ions of AZ obtained for the 13 experiments in Table 5. More than one chromatographic peak appeared in all cases, except for m/z 202 apparently, with apparent shifting in the 
retention times from experiment to experiment when orange extracts were injected (dotted lines). There were shifts with respect to the standards (continuous lines in Fig. 3) too. For

Like in Section 4.1, data tensors were built up for PARAFAC decomposition in order to calculate the experimental design responses. However, when complex matrix are analysed, as in this case, it has been proved [40] that the estimations of three-way models are more precise if both standards and fortified samples are included in the decomposition step. In this case, the 7 low level concentration calibration standards described in Section 2.4.1 were analysed (the sample containing $10 \mu \mathrm{L}^{-1}$ of each triazine was 5 times replicated) and the corresponding matrices were arranged together with those of the fortified samples of the experimental design into data tensors in such a way that a data tensors with dimensions $I \times 5 \times 25$ were obtained for each analyte. The first dimension of the datasets refers to the number of scans (I was 31, 22, 28, 26, 15, 10, 20 and 15 for SZ, AZ, PZ, TZ, ST, AT, PT and TT respectively), the second to the number of ions monitored, and the third to the number of samples (7 standards +5 replicated standard +13 experiments of the D-optimal design). A PARAFAC model was built for each analyte (the non-negativity constraint was enforced for the three ways), but the changes in the retention time between samples caused the PARAFAC models to fail. From the loadings of chromatographic and spectral modes, the relative retention time of no factor matched that of the reference standard and the relative ion abundances were not within the tolerance intervals established in ref. [13], i.e. the PARAFAC decomposition was not capable of successfully extracting the contribution of the signals corresponding to each triazine. This was due to the fact that PARAFAC model assumes that the chromatographic profiles do not change shape in different samples and the model fitted is highly affected if the structure of the data deviates considerably from the trilinear structure.

To solve this problem, PARAFAC2 models were built for each triazine and the internal standard by applying the ALS algorithm with unimodality and non-negativity constraints in the chromatographic mode and non-negativity constraint in spectral and sample modes, respectively. One important difference as against PARAFAC is that constraints in the first mode do not apply to the estimated profiles, $\mathbf{A}_{k}$, themselves but only to $\mathbf{H}$ (Eq. (3)). Although it is generally advised not to use constrains in the chromatographic mode, successful results were obtained by using them.

Table 6 summarizes the characteristics of the PARAFAC2 models built for this second Doptimal design for the optimization of QuEChERS procedure. The final models were chosen by comparing models with different number of factors, taking into account the explained variance, the CORCONDIA index, the unequivocal identification of each triazine (verification of compliance with the maximum permitted tolerances for the relative abundances and with the relative retention time tolerances), and the degree of agreement of the loadings of the sample mode. The CORCONDIA index is a measure of the degree of trilinearity of the data tensor and was developed by Bro and Kiers [41]; values over 70 are adequate. In the case of PT, the initial PARAFAC2 decomposition of the data tensor led to models with a CORCONDIA index far below 70 due to high interferent effect of co-eluents, which suggested that the trilinearity assumption was not fulfilled. In this case, the method required alignment to facilitate a suitable decomposition in the final model, with an adequate CORCONDIA value. The alignment was readily achieved by using the TIC, and next a smaller window of scans centred on the retention time of PT to avoid as far as possible coeluents was considered. 
Three-factor models were necessary in the PARAFAC2 decomposition for SZ, AZ, TZ and TT; for PZ, ST and PT, a two-factor model was necessary and a one-factor model for AT. In all the cases, the explained variances were larger than $96.5 \%$. In addition, the CORCONDIA index was always greater than $83 \%$, which indicated that the trilinearity hypothesis was assumable. The detection of outliers was carried out by calculating $Q$ residual and Hotelling's $T^{2}$ indices and removing those objects whose values exceed the corresponding threshold at a given confidence level. Only experiment 13 in Table 5 was considered as an outlier in the model built for PZ since those two indices exceeded their threshold values at the 99\% confidence level; for that reason this object was removed from the data tensor in the final PARAFAC2 decomposition for the internal standard.

The factor related to each triazine in the corresponding model was confirmed provided the pesticides were identified according to the requirements established in document SANCO/12495/2011 [13], as previously. Table 4 shows the relative abundances calculated with the loadings of the spectral profiles. The number of diagnostic ions that verified the compliance is shown in Table 6. At least 3 diagnostic ions verified the compliance in all cases, as SANCO/12495/2011 establishes for unequivocal identification of a compound. In cases were the fourth diagnostic ion did not verified the compliance, it was very close to the corresponding interval.

The loadings of spectral, chromatographic and sample modes obtained for AZ are showed in Fig. 4 as an example (the loadings of the chromatographic mode in PARAFAC2 models are referred throughout the paper to loadings scaled by the last mode loadings [28]). The loadings of the first factor were coherent with AZ, which enabled the unequivocal identification of this triazine (Fig. 4a shows, through the chromatographic profiles, how the shift in the retention time was pronounced). The loadings of the spectral mode of this factor (Fig. 4b) matched the spectra obtained from the spectrum of a $10 \mu \mathrm{g} \mathrm{L}^{-1}$ standard used as reference. And it was also confirmed that the relative retention time of the chromatographic profile obtained for each sample was within the tolerance intervals estimated from the reference sample. This means that the PARAFAC2 decomposition was capable of suitably extracting and differentiating the information related to AZ and co-eluents, which were related to spectral and chromatographic profiles of factors 2 and 3. As it can be clearly seen in the estimated sample profile, the loadings of the first factor increased with the concentration of AZ as expected for the first 12 samples (standards) in Fig. 4c, whereas those for the other two factors remained nearly constant for these samples and increased for orange extracts.

The loadings of the sample mode estimated for the internal standard (PZ) are shown in Fig. 5a. The second factor was identified as that the one related to PZ and the first corresponded to a co-eluent, which only had significant loadings for the orange extracts. The loadings obtained for the internal standard had practically constant values for the standards (samples from 1 to 12) and higher values for the oranges extracts. This was due to the matrix-induced response enhancement effect (co-extracts fill active sites, causing higher analyte transfer efficiency and thus greater signals in the presence of the matrix [42]), which is reduced by the use of the PTV injector but not completely eliminated [43]. This matrix-induced enhancement was observed for the chromatographic peaks of all triazines when orange extracts were injected.

Fig. 5b shows the standardized loadings of the sample mode calculated for AZ by dividing each loading of Fig. 4c by the corresponding loading of Fig. 5a. In this case, as well as compensating for small fluctuations in injection volume and changes in detector response, the use of internal standard corrected to a certain extent the effect on the loadings of the matrix- 
induced enhancement (this will be even clearer below, in Section 4.3). As the whole mass spectra and chromatographic peak were taken into account to calculate the loadings of both the analyte and the internal standard, the correction might be more effective [39].

601

602

603

604

605

The standardized loadings calculated for the 7 triazines were the responses for the D-optimal experimental design being performed for optimizing the QuEChERS procedure (see Table 5). Since experiment 13 was an outlier removed from the data set for PZ, therefore the final Doptimal design had only 12 experiments. This implied that the VIFs of the coefficients of the model of Eq. (6) ranged from 1.20 to 1.42, values which estimates precise enough guaranteed; the removing of one experiment of the experimental plan did not worsen significantly the quality of the estimates.

Due to high variability of the replicates and since the models were not significant at 95\% confidence level (only two models were significant at 90\%), in this case the statistical analysis based on the statistical significance of the models was not useful. As in addition the design was almost saturated since there are only 9 different experiments to estimate 8 coefficients, the interpretation of the size of the coefficients was more suitable. Fig. 6 shows the graphic study of the effects of the experimental factors studied on the responses.

In general, the effects of the experimental factors followed similar patterns for all the triazines. Taking into account these patterns all the factors were set at the corresponding high level for obtaining the largest responses. The vortex mixing time (mix_t1), the centrifugation time (centr_t1) and the percentage of acetic acid in acetonitrile (modifier) of the extraction step were $2 \mathrm{~min}, 5 \mathrm{~min}$ and $5 \%$ respectively; the volume of the extract (volume) and the mixing time (mix_t2) of the clean-up step were $1.2 \mathrm{~mL}$ and $1 \mathrm{~min}$; and the evaporation temperature (evap_T) was $40^{\circ} \mathrm{C}$. These levels are in solid bars in Fig. 6 .

\subsection{Analysis of samples: identification and quantification of triazines in oranges.}

Once the analytical procedure was optimized, the analysis of pesticides in orange samples was dealt with. The "second-order advantage" makes PARAFAC2 decomposition especially useful for quantifying and identifying analytes in complex samples where unknown interferents are present, as it has been seen above as it is the case here. This is of great interest in identifying and quantifying substances for which a permitted limit has been established (EU established MRLs for these pesticides: $0.10 \mathrm{mg} \mathrm{kg}^{-1}$ for TZ and $0.05 \mathrm{mg} \mathrm{kg}^{-1}$ for AZ [1]; and $0.01 \mathrm{mg} \mathrm{kg}^{-1}$ for SZ [26], ST, AT, PT and TT [27]).

European guidelines recommend the use of matrix-matched standards whenever matrixenhancement is demonstrated to minimize errors associated with it [44]. Therefore in addition to standards in ethyl acetate, matrix-matched standards which had the same concentration of co-extracted matrix components were also used in this analysis for building the PARAFAC2 models. In this case, a data tensor of dimension $\mathrm{I} \times 5 \times 44$ was obtained for every triazine after baseline correction. The first dimension of the data tensors refers to the number of scans (see $4^{\text {th }}$ column in Table 6), the second dimension to the number of diagnostic ions acquired for each compound, and the third dimension to the number of samples. The first 12 samples of the data tensor correspond to the 7 standards in Section 2.4.1 with concentrations from 0 to 20 $\mu \mathrm{g} \mathrm{L}{ }^{-1}$, plus 5 replicates of the $10 \mu \mathrm{g} \mathrm{L}^{-1}$ standard. The next 6 samples correspond to the fortified orange samples in Section 2.4.3 for low level concentration analysis; the first sample was a blank sample and the next 5 were fortified orange samples for recovery studies. The next 16 samples correspond to the 4 samples of Section 2.4.2, whose analysis was performed 
in quadruplicate. And finally, the last 10 samples correspond to the 10 matrix-matched standards in Section 2.4.4.

PARAFAC2 models were built from the decomposition of these data tensors; the characteristics of these models are summarized in Table 6. In some cases the final models have 43 samples instead of 44 because some outlier data were found. Chromatographic peaks of 3 and $10 \mu \mathrm{g} \mathrm{L}^{-1}$ matrix-matched standards were outliers for ST and TT respectively and they were removed from the corresponding data tensor. On the other hand, in the models built for TZ, AT and PT, $Q$ residual and Hotelling's $T^{2}$ indices which exceeded their threshold values at the $99 \%$ confidence level were obtained for the $20 \mu \mathrm{g} \mathrm{L}^{-1}$ matrix-matched standard, so this sample was removed from their data tensors and the three PARAFAC2 models were built again. In the case of PT, the method still required alignment of the chromatographic peaks.

The PARAFAC2 models had the same number of factors as (or greater than) the models built from standards and fortified orange samples in Section 4.2 (first rows in Table 6). This can be due to the fact that orange samples from different suppliers were arranged into the data tensor, so different co-eluents related to new factors could appear in some chromatographic peaks. In addition, these models had similar explained variance percentages and higher CORCONDIA indexes than the previous ones. Probably, the adding up of matrix-matched standards to the data tensors increased the trilinear structure of the data, what made the CORCONDIA indexes increased.

In all the PARAFAC models built for the triazines, except for AT, the presence of other coeluting substances was observed, i.e. GC/MS signals were non-specific, and for that reason more than one factor was necessary. In most cases this meant that the identification of the triazines according to the legislation could not be done either through the chromatogram or through the spectrum. The maximum permitted tolerances for the qualifier ions were exceeded in many cases when the analysis was carried out in the "usual way", taking into account the abundance acquired at a certain retention time. But this problem was avoided when the PARAFAC2 decomposition was used, leading to the unequivocal identification of all the triazines according to the requirements of legislation in force regarding both the relative retention time tolerances and the maximum permitted tolerances (see Table 4 ) for the relative ion abundance.

Again by way of example, a detailed analysis of the model built for AZ is shown. Fig. 7 shows the loadings obtained with the four-factor model. As in the previous case, the first factor was unequivocally identified as AZ, through the loadings of the chromatographic and spectral modes in Figs. 7a and $7 b$ (see the verification of compliance in Table 6). The impossibility of doing the identification by the "usual way" was clear in these figures. The interference of co-eluents were highly significant in Fig. 7b, so it was very unlikely that the corresponding relative abundances were within the maximum permitted tolerance intervals for the 3 diagnostic ions stated by the legislation. In addition, it was also probable that any coeluent would modify severely the relative retention time. In any case, this would have led to the wrong conclusion that there was not $\mathrm{AZ}$ in the sample, i.e. to false negatives.

The loadings of the sample mode for the first factor, Fig. 7c, followed the expected pattern; the higher the concentration of AZ the higher the values were obtained. Only the first factor had non null values of the loadings for standards (samples 1 to 7), which means that the rest of factors were related to other co-eluents of the extract and they only had non null values in the rest of samples (from extracts). Next, in the samples for recovery studies (8 to 13), the 
loadings of the first factor reflected the first blank sample (sample 8) and the next five 698 fortified samples (samples 9 to 13) perfectly; they took zero value for the first object and 699 higher values for the rest. Next, for the orange samples whose concentration was being determined (samples 14 to 34), the loadings had so low values that either there was no AZ in the samples or it was at very low concentrations. And finally, for the matrix-matched standards, the loadings increased their values with the concentration of $\mathrm{AZ}$, as expected, but higher values were obtained when comparing to samples 1 to 7 due to the matrix-induced enhancement. However, this effect was compensated if standardized loadings were considered; see Fig. 7d.

Once standardized loadings for the sample mode were calculated for all the triazines, calibration lines "standardized loading vs. concentration" were built with the matrix-matched standards. Table 7 shows the parameters of the regression models and the concentrations estimated for the orange samples of Section 2.4.2 together with the confidence intervals at a 95\% confidence level. The IUPAC recommendation has been followed; negative values of calculated concentration were neither substituted by zero nor removed. Most of the calculated concentrations for the samples of oranges were negative or statistically equal to zero (confidence intervals contains zero), and in those cases where a significant positive result was obtained, some of the values found were below the corresponding decision limits and all of them were far below of the corresponding MRLs. That is, no MRL violations were found.

\subsection{Figures of merit of the analytical procedure}

Some figures of merit such as accuracy (trueness and precision), recovery and repeatability, limit of decision and capability of detection both at null concentration and at the MRL were calculated.

\subsubsection{Accuracy: trueness and precision}

In order to determine trueness, a least squares regression line between the concentration calculated with the calibration model, $c_{\text {calc }}$, and the known concentration of the matrixmatched standards, $c_{\text {true }}$, was fitted. Outlier data were detected using the least median of squares (LMS) regression and then removed if their absolute values of standardized residual were higher than 2.5, in such a way that a reweighted least squares (RLS) regression model was built with the rest of data. Table 8 shows the parameters of the RLS models together with the number of standards, the calibration range (those which includes concentration equal to zero correspond to the quantification of the triazines in oranges) and some figures of merit. In order to compare the results obtained, the data corresponding to the standards (in ethyl acetate) are also included in the table. Similar results were obtained in both cases; despite small differences between the values of the standard deviation of regression $\left(\mathrm{s}_{\mathrm{yx}}\right)$ can be found. The precision of an analytical procedure can be estimated for the studied concentration range from the residual deviation standard of the regression " $c_{\text {calc }}$ vs. $c_{\text {true }}$ "; this value can be regarded as an estimate of the intermediate repeatability in the analysed concentration range [45]. Table 8 also shows the $\mathrm{s}_{\mathrm{yx}}$ values estimated for the different triazines.

But to guarantee trueness, the joint hypotheses "the slope is 1 and the intercept is zero" has to be jointly checked. Fig. 8 shows the joint confidence regions for slope and intercept estimated for both matrix-matched standards and standards. In all the cases, the analytical procedures fulfilled the property of trueness because the confidence ellipse contained the point $(0,1)$. The ellipse with the smallest size corresponded to PT in both cases (figs. 8(a) and 8(b)), and the size of the remaining ellipses varied depending on the standard deviation of each regression line. No significant differences were found between matrix-matched standards and standards. 
The differences observed in the orientation of the ellipses were only due to the number of data used to build the regression models.

In addition, Table 8 shows the mean of the absolute value of the relative errors in calibration. As expected, in all cases larger errors were obtained for the matrix-matched standards, but always within an acceptable range.

\subsubsection{Recovery}

Recovery was calculated from the 6 fortified orange samples (samples from 13 to 18 in the PARAFAC2 decomposition in Section 4.3) whose pretreatment was described in Section 2.4.3. The first sample was a blank sample which only contained the internal standard and the next 5 were orange samples fortified to contain $10 \mu \mathrm{g} \mathrm{L}{ }^{-1}$ of the 7 analysed triazines. In Table 8 , the average recovery rate is expressed as the percentage of the amount of each triazine initially added in each sample that was found with the analytical procedure. The found recovery rates ranged from $57 \%$ to $83 \%$, except for SZ, for which the recovery only reached $36.7 \%$. It is possible that the commercial kit used in the pretreatment step was not the most suitable for SZ.

\subsubsection{Repeatability}

This figure of merit was calculated as the standard deviation of the concentration calculated for the 5 samples fortified at $10 \mu \mathrm{g} \mathrm{L}^{-1}$ (samples from 14 to 18 of the PARAFAC2 decomposition in Section 4.3) for the orange samples, and from the 5 replicates of the standard of $10 \mu \mathrm{g} \mathrm{L}^{-1}$ (standards from 8 to 12 ). Values between 0.11 and $1.9 \mu \mathrm{g} \mathrm{L}^{-1}$ were obtained; higher values were also obtained for the fortified samples, as can be seen in Table 8 . This is reasonable taking the pretreatment step after the fortification of these samples into account; this increases the uncertainty of the analytical results.

\subsubsection{Decision limit and capability of detection}

According to ISO 11843 [46] the decision limit, $\mathrm{CC} \alpha$, is "the value of the net concentration the exceeding of which leads, for a given error probability $\alpha$, to the decision that the concentration of the analyte in the analysed material is larger than that in the blank material". Whereas the capability of detection, $x_{d}$ or CC $\beta$, for a given probability of false positive $\alpha$, is "the true net concentration of the analyte in the material to be analysed which will lead, with probability $1-\beta$, to the correct conclusion that the concentration in the analysed material is larger than that in the blank material". The need of assessing both the probability of false positive, $\alpha$, and of false negative, $\beta$, has also been recognized by IUPAC [47].

In multivariate or multi-way analysis, the decision limit and the capability of detection can be calculated from slope, intercept and $\mathrm{s}_{\mathrm{yx}}$ of the regression " $c_{\text {calc }}$ vs. $c_{\text {true }}$ " $[48,49]$, using the following equations:

$$
\mathrm{CC} \alpha=\frac{\mathrm{t}_{\alpha, \mathrm{N}-2} w_{\mathrm{x}_{0}} \hat{\sigma}}{\hat{\mathrm{b}}}
$$

$$
\operatorname{CC} \beta=\frac{\Delta(\alpha, \beta) w_{\mathrm{x}_{0}} \hat{\sigma}}{\hat{\mathrm{b}}}
$$


where $\Delta(\alpha, \beta)$ is the non-central parameter of a non-central Student's $t$-distribution related to the probabilities $\alpha$ and $\beta, w_{\mathrm{x}_{0}}$ is a parameter related to the distribution of the matrix-matched

standards on the $\mathrm{x}$-axis, and $\hat{\sigma}$ and $\hat{\mathrm{b}}$ are the standard deviation of regression and the slope of the regression " $c_{\text {calc }}$ vs. $c_{\text {true }}$ " respectively.

The decision limit and the detection capability were calculated for each triazine with probabilities of false positive, $\alpha$, and false negative, $\beta$, equal to 0.05 , and considering only one replicate. Table 8, column 11, shows the values of CC $\alpha$ obtained, which ranged from 0.51 to $1.15 \mu \mathrm{g} \mathrm{kg}^{-1}$. Decision limits did not differ very much for the different triazines, neither between standards nor for matrix-matched standards. The same can be concluded for detection capability, column 12, which ranged from 0.99 to $2.21 \mu \mathrm{g} \mathrm{kg}^{-1}$. In addition, the decision limit values are close to the detection capability values both in standards and in matrix-matched standards. Besides, all the values are far bellow the MRLs established for these triazines.

However since these triazines have MRLs, it is mandatory to calculate these figures of merit at the MRLs too. The MRL for SZ, ST, AT, PT and TT is $10 \mu \mathrm{gg}^{-1}$, so the calculation of the decision limit and detection capability of these triazines can be made from the regression models " $c_{\text {calc }}$ vs. $c_{\text {true }}$ " previously built provided that the calibration range includes the MRL (in the case of ST and AT in the matrix-matched standards, new regression models were built in the ranges from 1 to $20 \mu \mathrm{g} \mathrm{L}^{-1}$ and from 1 to $15 \mu \mathrm{g} \mathrm{L} \mathrm{L}^{-1}$ respectively; whose parameters are shown in Table 8). The values of CC $\alpha$ and CC $\beta$ at the MRL for these five triazines are detailed in Table 8. For SZ, for example, a value of CC $\beta$ of $11.51 \mu \mathrm{g} \mathrm{kg}^{-1}$ was obtained, which means that the analytical procedure is capable to distinguish $11.51 \mu \mathrm{g} \mathrm{kg}^{-1}$ from $10 \mu \mathrm{g}$ $\mathrm{kg}^{-1}$ with probabilities of false non-compliance and false compliance equal to 0.05 . CC $\alpha$ values are very close to the MRL, and CC $\beta$ values are very close to $C C \alpha$ values for each triazine, both in standards and in matrix-matched standards (see last two columns in table 8).

The MRLs of $\mathrm{AZ}$ and $\mathrm{TZ}$ are 50 and $100 \mu \mathrm{g} \mathrm{kg}$, they are far above the range of concentrations of the previous regression models so these cannot be used to estimate these figures of merit. For that reason, in order to estimate CC $\alpha$ and CC $\beta$ at the MRL the 7 high level concentration standards of Section 2.4.1, which contained $75 \mu \mathrm{g} \mathrm{L}^{-1}$ of the internal standard plus 20, 30, 40, 50, 60, 70 and $80 \mu \mathrm{g} \mathrm{L}^{-1}$ of AZ and 70, 80, 90, 100, 110, 120 and $130 \mu \mathrm{g} \mathrm{L^{-1 }}$ of $\mathrm{TZ}$, respectively, were introduced into the chromatographic system. The GC/MS signals were acquired together with the signals of five replicates of the standard which contained $50 \mu \mathrm{g} \mathrm{L}{ }^{-1}$ of $\mathrm{AZ}$ and $100 \mu \mathrm{g} \mathrm{L} \mathrm{L}^{-1}$ of $\mathrm{TZ}$, and the 6 samples corresponding to the fortified orange samples in Section 2.4.3 for high level concentration analysis.

After baseline correction, these signals were arranged in a data tensor with dimensions $\mathrm{I} \times 5 \times 18$ for each chromatographic peak. I refers to the number of scans and was 26, 26 and 27 for AZ, TZ and PZ respectively. The second dimension refers to the number of ions acquired for each compound; those specified in Section 2.4. And the third dimension is the number of samples of the data tensor: the first 7 samples corresponded to the 7 standards, samples 8 to 12 corresponded to the replicates of one of these standards, and samples 13 to 18 to the 6 fortified orange samples (the first sample was a blank sample and the next 5 were fortified orange samples). These last six samples provided information about the matrix-matched enhancement to the system for a more reliable PARAFAC2 decomposition. 
Fig. 1c shows the TIC acquired for one of these last samples, where the magnitude of the coeluents' peaks with respect to those of triazines is far lower than in Fig. 1(b). In fact, only one factor was necessary in the three PARAFAC2 decompositions carried out (the models built for AZ, TZ and PZ explained a 99.05, 99.63 and 99.17\% of variance). This meant that, at these concentration levels, the interferences of coeluents on the signals of triazines were unimportant, neither for $\mathrm{AZ}$ and $\mathrm{TZ}$ nor for the internal standard.

The relative ion abundances of the diagnostic ions were in all the cases within the permitted tolerance intervals and also the relative retention time corresponded to that of a reference sample (50 $\mu \mathrm{g} \mathrm{L}^{-1}$ of AZ, $100 \mu \mathrm{g} \mathrm{L}^{-1}$ of TZ and $75 \mu \mathrm{g} \mathrm{L}^{-1}$ of PZ) with a tolerance of $\pm 0.5 \%$, therefore mass spectral and chromatographic profiles of the unique factor were unequivocally identified. Next, the loadings of the sample profile were standardized by dividing each loading by the corresponding loading of PZ, and a calibration line between them and the concentration of each triazine was built. And finally, a regression model " $c_{\text {calc }}$ Vs. $c_{\text {true }}$ " was fit; the corresponding parameters are summarized in Table 8.

From these last models, like in the previous case, the decision limit (for a probability of false non-compliance, $\alpha$, equal to 0.05 ) and the capability of detection (for probabilities of false noncompliance, $\alpha$, and false compliance, $\beta$, equal to 0.05 ) at the MRL were calculated. The obtained values for $\mathrm{AZ}$ and $\mathrm{TZ}$ are shown in Table 8. Also in this case, $\mathrm{CC} \alpha$ values are very close to the MRLs, and CC $\beta$ values to $\mathrm{CC} \alpha$ values for both AZ and TZ. Although these figures of merit were not available in matrix-matched standards, the results obtained for the rest of triazines indicated that values close to those calculated for standards would be obtained also in matrix-matched standards.

\section{Conclusions}

The use of D-optimal designs in the optimizations steps have meant an important saving in the optimization cost, since the number of experiments were reduced from 384 to 16 in the first case and from 96 to 10 in the second one.

The QuEChERS procedure used caused relatively dirty extracts, which interfere in the quantification and in the unequivocal identification according to legislation in force for the analysis of triazines in oranges. Nevertheless, the problems with co-eluting interferents and with shifts in the retention time have been solved taking into account the three-way structure of the GC/MS data and using the PARAFAC2 decomposition (since it is less restrictive than PARAFAC decomposition).

Suitable validation results have been obtained for the analytical procedure proposed. No MLR violations have been found in the commercial oranges analysed.

\section{Acknowledgements}

The authors thank the financial support provided by projects of Ministerio de Economía y Competitividad (CTQ2011-26022) and Junta de Castilla y León (BU108A11-2).

\section{References}


[1] Commission Regulation (EC) No. 149/2008 of 29 January 2008 amending Regulation (EC) No. 396/2005 of the European Parliament and of the Council by establishing Annexes II, III and IV setting maximum residue levels for products covered by Annex I thereto. Official Journal of the European Union. L58, 1.3.2008, p. 1.

[2] D. Barceló, A.R. Fernández-Alba, C.L. Wilson (Eds.), Chromatographic-mass spectrometric food analysis for trace determination of pesticide residues, in Wilson \& Wilsoon's comprehensive analytical chemistry, Vol. 43, Elsevier, Amsterdam, 2005.

[3] M. Anastassiades, S.J. Lehotay, D. Stajnbaher, F.J. Schenck, J. AOAC Int. 86 (2003) $412 \mathrm{~A}$.

[4] P. Payá, M. Anastassiades, D. Mack, I. Sigalova, B. Tasdelen, J. Oliva , A. Barba, Anal. Bioanal. Chem. 389 (2007) 1697.

[5] S.J. Lehotay, K.A. Son, H. Kwon, U. Koesukwiwat, W. Fu, K. Mastovska, E. Hoh, N. Leepipatpiboon, J. Chromatog. A 1217 (2010) 2548.

[6] A. Wilkowska, M. Biziuk, Food Chem. 125 (2011) 803.

[7] G. Liu, L.Rong, B. Guo, M. Zhang, S. Li, Q. Wu, J. Chen, B. Chen, S. Yao, J. Chromatog. A 1218 (2011) 1429.

[8] R. Pereira Lopesa, R. Cazorla Reyes, R. Romero-González, J.L. Martínez Vidal, A. Garrido Frenich, J. Chromatog. B 895-896 (2012) 39.

[9] R. Pérez-Burgos, E.M. Grzelak, G. Gokce, J. Saurina, J. Barbosa, D. Barrón, J. Chromatog. B 899 (2012) 57.

[10] B. Kinsella, S.J. Lehotay, K. Mastovska, A.R. Lightfield, A. Furey, M. Danaher, Anal. Chim. Acta 637 (2009) 196.

[11] C. García Pinto, M.E. Fernández Laespada, S. Herrero Martín, A.M. Casas Ferreira, J.L. Pérez Pavón, B. Moreno Cordero, Talanta 81 (2010) 385.

[12] F. Plössl, M. Giera, F. Bracher, J. Chromatogr. A 1135 (2006) 19.

[13] Document N SANCO/12495/2011, Method Validation and Quality Control Procedures for Pesticide Residues Analysis in Food and Feed. (2011) EU, Brussels.

[14] L. Rubio, L.A. Sarabia, A. Herrero, M.C. Ortiz, Anal. Bional. Chem. 403-4 (2012) 1618.

[15] M.C. Ortiz, L.A. Sarabia, J. Chromatogr. A 1158 (2007) 94.

[16] J.M. Amigo, T. Skov, J. Coello, S. Maspoch, R. Bro, Trends Anal. Chem. 27 (2008) 714.

[17] B.D. Real, M.C. Ortiz, L.A. Sarabia, J. Chromatogr. B 910 (2012) 122.

[18] J.M. Amigo, M.J. Popielarz, R.M. Callejón, M.L. Morales, A.M. Troncoso, M.A. Petersen, T.B. Toldam-Andersen, J. Chromatogr. A 1217 (2010) 4422.

[19] R. Morales, M.C. Ortiz, L.A. Sarabia, Anal. Chim. Acta 754 (2012) 20.

[20] H.G.J. Mol, M. Althuizen, H.G. Janssen, C.A. Cramers, J. High Resol. Chromatogr. 19 (1996) 69.

[21] P.F. De Aguiar, B. Bourguignon, M.S. Khost, D.L. Massart, R. Phan-Tan-Luu, Chemom. Intell. Lab. Systems 30 (1995) 199. 
[22] G.A. Lewis, D. Mathieu, R. Phan-Tan-Luu, Pharmaceutical and Experimental Designs, Marcel Dekker, New York, 1999.

[23] D. Arroyo, M.C. Ortiz, L.A. Sarabia, J. Chromatog. A 1218 (2011) 4487.

[24] N. Rodríguez, M.C. Ortiz, L.A. Sarabia, A. Herrero, Anal. Chim. Acta 657 (2010) 136.

[25] N. Li, L. Nian, R. Zhang, S. Wu, R. Ren, Y. Wang, H. Zhang, A. Yu, Talanta 105 (2013) 219.

[26] Commission Regulation (EU) No. 310/2011 of 28 March 2011 amending Annexes II and III to Regulation (EC) No 396/2005 of the European Parliament and of the Council as regards maximum residue levels for aldicarb, bromopropylate, chlorfenvinphos, endosulfan, EPTC, ethion, fenthion, fomesafen, methabenzthiazuron, methidathion, simazine, tetradifon and triforine in or on certain products. Official Journal of the European Union L86, 1.4.2011, p. 1.

[27] Regulation (EC) No. 396/2005 of the European Parliament and of the Council of 23 February 2005 on maximum residue levels of pesticides in or on food and feed of plant and animal origin and amending Council Directive 91/414/EEC. Official Journal of the European Union L70, 16.3.2005, p. 1.

[28] B.M. Wise, N.B. Gallagher, R. Bro, J.M. Shaver, W. Windig, R.S. Koch, PLS Toolbox 5.8.2. Eigenvector Research Inc., Manson, WA, 2010.

[29] STATGRAPHICS Centurion XVI (version 16.1.11), StatPoint Technologies, Inc. Herndon, VA, 2010.

[30] P.J. Rousseeuw, A.M. Leroy, Robust Regression and Outliers Detection, John Wiley and Sons, New Jersey, 2001.

[31] L.A. Sarabia, M.C. Ortiz, TrAC: Trends Anal. Chem. 13 (1994) 1.

[32] D. Mathieu, J. Nony, R. Phan-Than-Lu, NemrodW (Version 2007_03), L.P.R.A.I. Marseille, France, 2007.

[33] C.A. Anderson, R. Bro, Chemom. Intell. Lab. Syst. 52 (2000) 1.

[34] R. Bro, Chemom. Intell. Lab. Syst. 46 (1999) 133.

[35] H.A.L. Kiers, J.M.F. Ten Berge, R. Bro, J. Chemom. 13 (1999) 275.

[36] R. Bro, C.A. Andersson, H.A.L. Kiers, J. Chemom. 13 (1999) 295.

[37] D. Arroyo, M.C. Ortiz, L.A. Sarabia, J. Chromatogr. A 1157 (2007) 358.

[38] B.D. Real, M.C. Ortiz, L.A. Sarabia, Talanta 71 (2007) 1599.

[39] I. García, L. Sarabia, M.C. Ortiz, J.M. Aldama, Anal. Chim. Acta 526 (2004) 139.

[40] M.C. Ortiz, L.A. Sarabia, I. García, D. Giménez, E. Meléndez, Anal. Chim. Acta 559 (2006) 124.

[41] R. Bro, H.A.L. Kiers, J. Chemometrics 17 (2003) 274.

[42] D.R. Erney, A.M. Gillespie, D.M. Gilvydis, C.F. Poole, J. Chromatogr. 638 (1993) 57.

[43] C.F. Poole, J. Chromatogr. A 1158 (2007) 241.

[44] CEN standard method EN 15662:2008. Foods of plant origin. Determination of pesticide residues using GC-MS and/or LC-MS/MS following acetonitrile extraction/partitioning and clean-up by dispersive SPE - QuEChERS method. 
[45] M.B. Sanz, L.A. Sarabia, A. Herrero, M.C. Ortiz, Talanta 56 (2002) 1039.

[46] International Organization for Standardization, ISO 11843, Capability of detection. Part 1: Terms and definitions, 1997; and Part 2: Methodology in the linear calibration case, 2000. Geneva, Switzerland.

[47] J. Inczédy, T. Lengyel, A.M. Ure, A. Gelencsér, A. Hulanicki, Compendium of Analytical Nomenclature IUPAC, Pot City Press Inc, Baltimore, 3rd ed., 2000.

[48] M.C. Ortiz, L.A. Sarabia, A. Herrero, M.S. Sánchez, M.B. Sanz, M.E. Rueda, D. Giménez, M.E. Meléndez, Chemom. Intell. Lab. Syst. 69 (2003) 21.

[49] M.C. Ortiz, M.S. Sánchez, L.A. Sarabia, in: S.D. Brown, R. Tauler, B. Walczak (Eds.), Comprehensive Chemometrics, Elsevier, Amsterdam, 2009, pp. 127. 
Table 1 Factors and experimental domain for optimization of the PTV injection.

\begin{tabular}{lcccc}
\hline \multicolumn{1}{c}{ Factors } & $\begin{array}{c}\text { Codified } \\
\text { variable }\end{array}$ & Level A & Level B & Level C \\
\hline Vent flow $\left(\mathrm{mL} \mathrm{min}^{-1}\right)$ & $\mathrm{x}_{1}$ & 100 & 150 & - \\
Inlet P $(\mathrm{psi})$ & $\mathrm{x}_{2}$ & 8 & 9 & - \\
Vent time $(\mathrm{min})$ & $\mathrm{x}_{3}$ & 0.3 & 0.45 & - \\
Initial temperature $\left({ }^{\circ} \mathrm{C}\right)$ & $\mathrm{x}_{4}$ & 40 & 50 & - \\
PTV initial time $(\mathrm{min})$ & $\mathrm{x}_{5}$ & 0.6 & 0.5 & - \\
PTV rate $\left({ }^{\circ} \mathrm{C} \mathrm{s}^{-1}\right)$ & $\mathrm{x}_{6}$ & 10 & 5 & - \\
End temperature $\left({ }^{\circ} \mathrm{C}\right)$ & $\mathrm{x}_{7}$ & 280 & 320 & - \\
Injection speed $\left(\mu \mathrm{L} \mathrm{s}{ }^{-1}\right)$ & $\mathrm{x}_{8}$ & 0.85 & 1.7 & 3.4 \\
\hline
\end{tabular}


Table 2 Factors and experimental domain for optimization of the QuEChERS procedure.

\begin{tabular}{lccccc}
\hline \multicolumn{1}{c}{ Factors } & $\begin{array}{c}\text { Abbreviated } \\
\text { name }\end{array}$ & $\begin{array}{c}\text { Codified } \\
\text { variable }\end{array}$ & Level A & Level B & Level C \\
\hline Time of vortex mixing 1 (min) & mix_t1 & $\mathrm{x}_{1}$ & 1 & 2 & - \\
Time of centrifugation (min) & centr_t1 & $\mathrm{x}_{2}$ & 1 & 5 & - \\
Acetonitrile extract volume $(\mathrm{mL})$ & volume & $\mathrm{x}_{3}$ & 1 & 1.2 & - \\
Time of vortex mixing 2 $(\mathrm{s})$ & mix_t2 & $\mathrm{x}_{4}$ & 30 & 60 & - \\
Evaporation temperature $\left({ }^{\circ} \mathrm{C}\right)$ & evap_T & $\mathrm{x}_{5}$ & 40 & 50 & - \\
Percent $(\mathrm{v} / \mathrm{v})$ of acetic acid in & modifier & $\mathrm{x}_{6}$ & 0 & 1 & 5 \\
acetonitrile $(\%)$ & & & & \\
\hline
\end{tabular}


Table 3 Experimental plan and responses of the D-optimal design for the optimization of the PTV injection.

\begin{tabular}{|c|c|c|c|c|c|c|c|c|c|c|c|c|c|c|c|}
\hline \multirow{2}{*}{ Run } & \multirow{2}{*}{$\begin{array}{l}\text { Vent flow } \\
\left(\mathrm{mL} \mathrm{m^{-1 }}\right)\end{array}$} & \multirow{2}{*}{$\begin{array}{c}\text { Inlet } \\
\mathrm{P} \\
\text { (psi) }\end{array}$} & \multirow{2}{*}{$\begin{array}{l}\text { Vent } \\
\text { time } \\
(\min )\end{array}$} & \multirow{2}{*}{$\begin{array}{c}\text { Initial } \\
\text { temperature } \\
\left({ }^{\circ} \mathrm{C}\right)\end{array}$} & \multirow{2}{*}{$\begin{array}{c}\text { PTV } \\
\text { initial } \\
\text { time } \\
(\mathrm{min})\end{array}$} & \multirow{2}{*}{$\begin{array}{l}\mathrm{PTV} \\
\text { rate } \\
\left({ }^{\circ} \mathrm{C} \mathrm{s}^{-1}\right)\end{array}$} & \multirow{2}{*}{$\begin{array}{c}\text { End } \\
\text { temperature } \\
\left({ }^{\circ} \mathrm{C}\right)\end{array}$} & \multirow{2}{*}{$\begin{array}{l}\text { Injection } \\
\text { speed } \\
\left(\mu \mathrm{L} \mathrm{s}^{-1}\right)\end{array}$} & \multicolumn{7}{|c|}{ Responses (standardized loadings) } \\
\hline & & & & & & & & & $\mathrm{SZ}$ & $\mathrm{AZ}$ & $\mathrm{TZ}$ & ST & AT & PT & $\mathrm{TT}$ \\
\hline 1 & 150 & 8 & 0.45 & 40 & 0.6 & 10 & 280 & 0.85 & 0.45 & 0.76 & 1.61 & 0.79 & 0.79 & 1.24 & 1.08 \\
\hline 2 & 150 & 8 & 0.30 & 50 & 0.6 & 5 & 280 & 0.85 & 0.43 & 0.74 & 1.60 & 0.75 & 0.76 & 1.19 & 1.07 \\
\hline 3 & 100 & 9 & 0.45 & 40 & 0.5 & 5 & 280 & 0.85 & 0.44 & 0.74 & 1.60 & 0.78 & 0.79 & 1.26 & 1.11 \\
\hline 4 & 100 & 9 & 0.45 & 50 & 0.6 & 10 & 320 & 0.85 & 0.48 & 0.77 & 1.63 & 0.95 & 0.91 & 1.42 & 1.24 \\
\hline 5 & 100 & 8 & 0.30 & 50 & 0.5 & 10 & 320 & 0.85 & 0.46 & 0.75 & 1.63 & 0.87 & 0.84 & 1.32 & 1.13 \\
\hline 6 & 100 & 9 & 0.30 & 40 & 0.5 & 5 & 320 & 0.85 & 0.47 & 0.77 & 1.60 & 0.90 & 0.87 & 1.34 & 1.17 \\
\hline 7 & 100 & 8 & 0.45 & 50 & 0.5 & 10 & 280 & 1.70 & 0.46 & 0.77 & 1.62 & 0.80 & 0.79 & 1.25 & 1.11 \\
\hline 8 & 100 & 8 & 0.30 & 40 & 0.6 & 5 & 280 & 1.70 & 0.46 & 0.76 & 1.60 & 0.85 & 0.82 & 1.28 & 1.13 \\
\hline 9 & 150 & 9 & 0.45 & 50 & 0.5 & 5 & 280 & 1.70 & 0.44 & 0.75 & 1.61 & 0.79 & 0.79 & 1.27 & 1.10 \\
\hline 10 & 150 & 9 & 0.45 & 40 & 0.6 & 10 & 320 & 1.70 & 0.50 & 0.79 & 1.62 & 0.93 & 0.87 & 1.35 & 1.19 \\
\hline 11 & 150 & 8 & 0.30 & 40 & 0.5 & 10 & 320 & 1.70 & 0.50 & 0.78 & 1.65 & 1.00 & 0.93 & 1.44 & 1.27 \\
\hline 12 & 150 & 8 & 0.30 & 40 & 0.5 & 10 & 320 & 1.70 & 0.47 & 0.79 & 1.59 & 0.89 & 0.85 & 1.29 & 1.12 \\
\hline 13 & 150 & 8 & 0.30 & 40 & 0.5 & 10 & 320 & 1.70 & 0.49 & 0.78 & 1.61 & 0.94 & 0.90 & 1.35 & 1.19 \\
\hline 14 & 150 & 8 & 0.30 & 40 & 0.5 & 10 & 320 & 1.70 & 0.49 & 0.78 & 1.64 & 0.97 & 0.93 & 1.40 & 1.25 \\
\hline 15 & 150 & 9 & 0.30 & 50 & 0.6 & 5 & 320 & 1.70 & 0.48 & 0.77 & 1.70 & 0.90 & 0.87 & 1.32 & 1.18 \\
\hline 16 & 100 & 9 & 0.30 & 50 & 0.6 & 10 & 280 & 3.40 & 0.46 & 0.77 & 1.63 & 0.86 & 0.83 & 1.31 & 1.16 \\
\hline 17 & 150 & 9 & 0.30 & 40 & 0.5 & 10 & 280 & 3.40 & 0.47 & 0.77 & 1.63 & 0.84 & 0.82 & 1.30 & 1.13 \\
\hline 18 & 100 & 8 & 0.45 & 40 & 0.6 & 5 & 320 & 3.40 & 0.50 & 0.79 & 1.63 & 0.95 & 0.89 & 1.36 & 1.19 \\
\hline 19 & 150 & 8 & 0.45 & 50 & 0.5 & 5 & 320 & 3.40 & 0.48 & 0.77 & 1.62 & 0.93 & 0.89 & 1.35 & 1.19 \\
\hline
\end{tabular}


Table 4 Detected ions (the most intense ones are in bold), relative abundance and tolerance intervals for the reference sample of $10 \mu \mathrm{g} \mathrm{L}^{-1}$ of the eight triazines, and relative abundances calculated with the loadings of the spectral profiles of the PARAFAC2 models built from both the samples of the D-optimal design of the QuEChERS procedure optimization and from the quantitative determination samples.

\begin{tabular}{|c|c|c|c|c|c|}
\hline \multirow{2}{*}{ Analyte } & \multirow{2}{*}{$\mathrm{m} / \mathrm{z}$} & \multicolumn{2}{|c|}{ Reference sample } & \multirow{2}{*}{$\begin{array}{c}\text { D-optimal design } \\
\text { samples } \\
\text { (QuEChERS) }\end{array}$} & \multirow{2}{*}{$\begin{array}{c}\begin{array}{c}\text { Quantitative } \\
\text { determination } \\
\text { samples }\end{array} \\
\text { Relative } \\
\text { abundance (\%) }\end{array}$} \\
\hline & & $\begin{array}{c}\text { Relative } \\
\text { abundance (\%) }\end{array}$ & $\begin{array}{c}\text { Tolerance } \\
\text { interval (\%) }\end{array}$ & & \\
\hline \multirow{5}{*}{$\mathrm{SZ}$} & 158 & 23.74 & $(20.18-27.30)$ & 27.25 & 29.33 \\
\hline & 173 & 44.55 & $(37.87-51.24)$ & 42.88 & 38.53 \\
\hline & 186 & 71.89 & $(64.70-79.08)$ & 81.85 & 66.79 \\
\hline & 201 & 100.00 & - & 100.00 & 100.00 \\
\hline & 203 & 31.95 & $(27.16-36.74)$ & 32.82 & 32.45 \\
\hline \multirow{5}{*}{$\mathrm{AZ}$} & 173 & 24.55 & $(20.86-28.23)$ & 25.39 & 21.09 \\
\hline & 200 & 100.00 & - & 100.00 & 100.00 \\
\hline & 202 & 33.86 & $(28.78-38.94)$ & 35.33 & 35.28 \\
\hline & 215 & 57.57 & $(51.82-63.33)$ & 51.66 & 53.42 \\
\hline & 217 & 19.07 & $(16.21-21.93)$ & 19.85 & 14.51 \\
\hline \multirow{5}{*}{$\mathrm{PZ}$} & 172 & 57.57 & $(51.81-63.33)$ & 52.75 & 52.07 \\
\hline & 187 & 26.51 & $(22.53-30.49)$ & 50.87 & 29.12 \\
\hline & 214 & 100 & - & 100.00 & 100.00 \\
\hline & 229 & 60.63 & $(54.56-66.69)$ & 56.45 & 50.44 \\
\hline & 231 & 19.36 & $(16.45-22.26)$ & 17.54 & 19.25 \\
\hline \multirow{5}{*}{$\mathrm{TZ}$} & 173 & 35.50 & $(30.17-40.82)$ & 36.22 & 33.84 \\
\hline & 214 & 100 & - & 100.00 & 100.00 \\
\hline & 216 & 31.03 & $(26.38-35.69)$ & 35.29 & 33.80 \\
\hline & 229 & 24.24 & $(20.60-27.87)$ & 23.12 & 24.26 \\
\hline & 231 & 7.40 & $(3.70-11.09)$ & 6.96 & 6.96 \\
\hline \multirow{5}{*}{ ST } & 155 & 33.88 & $(28.80-38.96)$ & 30.19 & 24.14 \\
\hline & 170 & 31.92 & $(27.13-36.71)$ & 34.27 & 32.86 \\
\hline & 198 & 18.44 & $(14.75-22.13)$ & 17.64 & 15.84 \\
\hline & 213 & 100.00 & - & 100.00 & 100.00 \\
\hline & 215 & 4.74 & $(2.37-7.11)$ & 5.04 & 5.08 \\
\hline \multirow{7}{*}{ AT } & 170 & 24.21 & $(20.58-27.84)$ & 26.95 & 32.60 \\
\hline & 185 & 18.04 & $(15.34-20.75)$ & 19.61 & 20.51 \\
\hline & 212 & 59.45 & $(53.50-65.39)$ & 56.47 & 60.84 \\
\hline & 227 & 100.00 & - & 100.00 & 100.00 \\
\hline & 229 & 5.53 & $(2.77-8.30)$ & 5.87 & 6.08 \\
\hline & 184 & 84.13 & $(75.71-92.54)$ & 91.02 & 87.39 \\
\hline & 199 & 26.22 & $(22.29-30.16)$ & 26.56 & 24.29 \\
\hline
\end{tabular}




\begin{tabular}{|c|c|c|c|c|c|}
\hline \multirow[t]{3}{*}{ PT } & 226 & 61.88 & $(55.69-68.06)$ & 65.90 & 63.44 \\
\hline & 241 & 100.00 & - & 100.00 & 100.00 \\
\hline & 243 & 5.12 & $(2.56-7.68)$ & 6.91 & 5.60 \\
\hline \multirow{5}{*}{ TT } & 170 & 57.20 & $(51.48-62.92)$ & 62.16 & 60.00 \\
\hline & 185 & 70.64 & $(63.58-77.71)$ & 69.97 & 68.62 \\
\hline & 226 & 100.00 & - & 100.00 & 100.00 \\
\hline & 241 & 53.46 & $(48.11-58.80)$ & 52.38 & 55.11 \\
\hline & 243 & 4.30 & $(2.15-6.45)$ & 1.57 & 4.91 \\
\hline
\end{tabular}


Table 5 Experimental plan and responses of the D-optimal design for the optimization of the QuEChERS procedure.

\begin{tabular}{|c|c|c|c|c|c|c|c|c|c|c|c|c|c|}
\hline \multirow{2}{*}{ Run } & \multirow{2}{*}{$\begin{array}{c}\operatorname{mix\_ t1} \\
(\mathrm{min})\end{array}$} & \multirow{2}{*}{$\begin{array}{c}\text { centr_t1 } \\
\text { (min) }\end{array}$} & \multirow{2}{*}{$\begin{array}{c}\text { volume } \\
(\mathrm{mL})\end{array}$} & \multirow{2}{*}{$\underset{(\mathrm{s})}{\operatorname{mix\_ t} t 2}$} & \multirow{2}{*}{$\begin{array}{c}\text { evap_T } \\
\left({ }^{\circ} \mathrm{C}\right)\end{array}$} & \multirow{2}{*}{$\begin{array}{c}\text { modifier } \\
(\%)\end{array}$} & \multicolumn{7}{|c|}{ Responses (standardized loadings) } \\
\hline & & & & & & & $\mathrm{SZ}$ & $\mathrm{AZ}$ & $\mathrm{TZ}$ & $\mathrm{ST}$ & AT & PT & TT \\
\hline 1 & 2 & 1 & 1.2 & 30 & 40 & 0 & 0.28 & 0.70 & 0.72 & 0.98 & 0.65 & 0.73 & 0.88 \\
\hline 2 & 1 & 1 & 1.0 & 60 & 40 & 0 & 0.34 & 0.70 & 0.96 & 0.89 & 0.97 & 0.89 & 0.72 \\
\hline 3 & 1 & 5 & 1.0 & 30 & 50 & 0 & 0.35 & 0.45 & 0.69 & 0.67 & 0.75 & 0.76 & 0.61 \\
\hline 4 & 1 & 5 & 1.0 & 30 & 50 & 0 & 0.26 & 0.34 & 0.53 & 0.53 & 0.59 & 0.59 & 0.38 \\
\hline 5 & 1 & 5 & 1.0 & 30 & 50 & 0 & 0.32 & 0.52 & 0.63 & 0.67 & 0.69 & 0.68 & 0.31 \\
\hline 6 & 1 & 5 & 1.0 & 30 & 50 & 0 & 0.38 & 0.84 & 0.93 & 1.11 & 0.91 & 1.06 & 0.78 \\
\hline 7 & 2 & 5 & 1.2 & 60 & 50 & 0 & 0.47 & 0.88 & 1.20 & 1.19 & 1.21 & 1.21 & 0.45 \\
\hline 8 & 1 & 5 & 1.2 & 30 & 40 & 1 & 0.44 & 0.97 & 1.08 & 1.20 & 0.99 & 1.10 & 0.56 \\
\hline 9 & 2 & 5 & 1.0 & 60 & 40 & 1 & 0.49 & 1.01 & 1.27 & 1.22 & 1.14 & 1.24 & 0.86 \\
\hline 10 & 2 & 1 & 1.0 & 30 & 50 & 1 & 0.44 & 0.81 & 1.04 & 1.02 & 1.00 & 1.04 & 0.50 \\
\hline 11 & 1 & 1 & 1.2 & 60 & 50 & 1 & 0.50 & 0.75 & 0.96 & 1.02 & 1.17 & 1.10 & 0.83 \\
\hline 12 & 2 & 5 & 1.0 & 30 & 40 & 5 & 0.50 & 0.81 & 1.18 & 1.23 & 1.07 & 1.25 & 1.08 \\
\hline 13 & 1 & 1 & 1.2 & 60 & 50 & 5 & - & - & - & - & - & - & - \\
\hline
\end{tabular}


Table 6 Characteristics of PARAFAC2 models: number of factors, data tensor size, explained variance, CORCONDIA index and verification of compliance with the maximum permitted tolerances for the identification of each triazine (number of diagnostic ions that are within the tolerance intervals calculated for each sample using a reference sample containing $10 \mu \mathrm{g} \mathrm{L}{ }^{-1}$ of SZ, AZ, PZ, TZ, ST, AT, PT, and TT).

\begin{tabular}{|c|c|c|c|c|c|c|}
\hline Study & Analyte & Factors & $\begin{array}{c}\text { Data tensor } \\
\text { dimension }^{\text {a }} \\
\mathrm{I} \times \mathrm{J} \times \mathrm{K}\end{array}$ & $\begin{array}{l}\text { Explained } \\
\text { variance } \\
(\%)\end{array}$ & $\begin{array}{l}\text { CORCONDIA } \\
\text { index } \\
(\%)\end{array}$ & $\begin{array}{c}\text { Verified } \\
\text { compliance }\end{array}$ \\
\hline \multirow{8}{*}{$\begin{array}{l}\text { D-optimal design } \\
\text { to optimize } \\
\text { QuEChERS } \\
\text { procedure }\end{array}$} & $\mathrm{SZ}$ & 3 & $31 \times 5 \times 25$ & 96.53 & 85.91 & 3 \\
\hline & $\mathrm{AZ}$ & 3 & $22 \times 5 \times 25$ & 99.28 & 83.61 & 3 \\
\hline & $\mathrm{PZ}$ & 2 & $28 \times 5 \times 24$ & 97.33 & 100.00 & 3 \\
\hline & $\mathrm{TZ}$ & 3 & $26 \times 5 \times 25$ & 99.43 & 86.56 & 4 \\
\hline & ST & 2 & $15 \times 5 \times 25$ & 99.32 & 100.00 & 3 \\
\hline & $\mathrm{AT}$ & 1 & $10 \times 5 \times 25$ & 98.96 & $-{ }^{\mathrm{b}}$ & 4 \\
\hline & $\mathrm{PT}$ & 2 & $6 \times 5 \times 25$ & 98.3 & 100 & 4 \\
\hline & $\mathrm{TT}$ & 3 & $15 \times 5 \times 25$ & 97.96 & 83.30 & 3 \\
\hline \multirow{8}{*}{$\begin{array}{c}\text { Quantitative and } \\
\text { qualitative } \\
\text { determination of } \\
\text { triazines in oranges }\end{array}$} & $\mathrm{SZ}$ & 3 & $28 \times 5 \times 44$ & 96.5 & 99.58 & 3 \\
\hline & $\mathrm{AZ}$ & 4 & $21 \times 5 \times 44$ & 98.76 & 97.86 & 3 \\
\hline & $\mathrm{PZ}$ & 2 & $28 \times 5 \times 44$ & 98.71 & 100.00 & 3 \\
\hline & $\mathrm{TZ}$ & 3 & $26 \times 5 \times 43$ & 98.67 & 90.78 & 4 \\
\hline & ST & 3 & $15 \times 5 \times 43$ & 99.17 & 99.67 & 3 \\
\hline & $\mathrm{AT}$ & 1 & $10 \times 5 \times 43$ & 97.4 & $-{ }^{b}$ & 3 \\
\hline & $\mathrm{PT}$ & 3 & $6 \times 5 \times 43$ & 99.97 & 98.8 & 4 \\
\hline & $\mathrm{TT}$ & 3 & $15 \times 5 \times 25$ & 98.5 & 99.07 & 4 \\
\hline
\end{tabular}

(a) I refers to the number of scans, J refers to the number of ions, and $\mathrm{K}$ refers to the number of samples

(b) It was not possible to calculate the CORCONDIA index as there was only one factor 
Table 7 Parameters of the calibration lines "standardized loadings vs. true concentration" (intercept, slope, standard deviation of regression $\left(\mathrm{s}_{\mathrm{yx}}\right)$ and correlation coefficient) and calculated concentrations, $\mathrm{c}_{\mathrm{cal}}$, for the four commercial orange samples $(95 \%$ confidence intervals are in brackets). The calculated concentrations are the mean of the four replicates analysed for each sample.

\begin{tabular}{|c|c|c|c|c|c|c|c|c|}
\hline & & $\mathrm{SZ}$ & $\mathrm{AZ}$ & $\mathrm{TZ}$ & ST & AT & $\mathrm{PT}$ & TT \\
\hline \multirow{4}{*}{$\begin{array}{l}\text { Regression } \\
\text { parameters }\end{array}$} & Intercept & 0.0459 & -0.0037 & 0.0793 & -0.0256 & 0.0198 & 0.1225 & 0.0685 \\
\hline & Slope & 0.0460 & 0.0823 & 0.1116 & 0.1122 & 0.0801 & 0.1387 & 0.1181 \\
\hline & $\mathrm{s}_{\mathrm{yx}}$ & 0.0172 & 0.0289 & 0.0259 & 0.0280 & 0.0249 & 0.0340 & 0.0596 \\
\hline & Correlation coefficient & 0.9989 & 0.9987 & 0.9939 & 0.9928 & 0.9911 & 0.9989 & 0.9978 \\
\hline \multirow{4}{*}{$\begin{array}{c}\mathrm{C}_{\text {calc }} \\
\left(\mu \mathrm{g} \mathrm{L}^{-1}\right)\end{array}$} & $\mathrm{P} 1$ & $\begin{array}{c}0.62 \\
(-0.01,1.23)\end{array}$ & $\begin{array}{c}1.14 \\
(0.61,1.65)\end{array}$ & $\begin{array}{c}0.27 \\
(-0.34,0.76)\end{array}$ & $\begin{array}{c}0.24 \\
(-0.35,0.73)\end{array}$ & $\begin{array}{c}1.16 \\
(0.58,1.67)\end{array}$ & $\begin{array}{c}-0.23 \\
(-0.64,0.16)\end{array}$ & $\begin{array}{c}-0.14 \\
(-0.98,0.66)\end{array}$ \\
\hline & $\mathrm{P} 2$ & $\begin{array}{c}0.00 \\
(-0.65,0.62)\end{array}$ & $\begin{array}{c}0.63 \\
(0.09,1.15)\end{array}$ & $\begin{array}{c}0.04 \\
(-0-59,0.55)\end{array}$ & $\begin{array}{c}0.26 \\
(-0.32,0.75)\end{array}$ & $\begin{array}{c}1.34 \\
(0.78,1.84)\end{array}$ & $\begin{array}{c}-0.34 \\
(-0.75,0.06)\end{array}$ & $\begin{array}{c}0.04 \\
(-0.79,0.83)\end{array}$ \\
\hline & $\mathrm{P} 3$ & $\begin{array}{c}0.15 \\
(-0.50,0.76)\end{array}$ & $\begin{array}{c}0.99 \\
(0.46,1.51)\end{array}$ & $\begin{array}{c}0.13 \\
(-0.49,0.63)\end{array}$ & $\begin{array}{c}0.24 \\
(-0.35,0.73)\end{array}$ & $\begin{array}{c}0.87 \\
(0.26,1.39)\end{array}$ & $\begin{array}{c}-0.6 \\
(-1.01,-0.20)\end{array}$ & $\begin{array}{c}0.19 \\
(-0.64,0.98)\end{array}$ \\
\hline & P4 & $\begin{array}{c}0.01 \\
(-0.64,0.63)\end{array}$ & $\begin{array}{c}1.24 \\
(0.72,1.76)\end{array}$ & $\begin{array}{c}0.01 \\
(-0.62,0.53)\end{array}$ & $\begin{array}{c}0.24 \\
(-0.34,0.73)\end{array}$ & $\begin{array}{c}1.20 \\
(0.63,1.71)\end{array}$ & $\begin{array}{c}-0.47 \\
(-0.88,-0.07)\end{array}$ & $\begin{array}{c}-0.01 \\
(-0.85,0.78)\end{array}$ \\
\hline
\end{tabular}




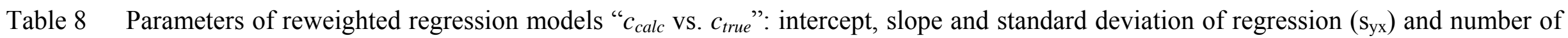
standards (n). Some figures of merit: error (mean of the absolute value of relative errors in calibration), average recovery rate, repeatability and detection limit $(C C \alpha)$ and capability of detection $(C C \beta)$ at $\mathrm{x}_{0}=0$ and at the maximum residue level $\left(\mathrm{x}_{0}=\mathrm{MRL}\right)$ for both standards and matrix-matched standards.

\begin{tabular}{|c|c|c|c|c|c|c|c|c|c|c|c|c|c|}
\hline & \multirow[b]{2}{*}{ Analyte } & \multirow{2}{*}{\multicolumn{2}{|c|}{$\mathrm{n} \quad \begin{array}{c}\text { Concentration } \\
\text { range } \\
\left(\mu \mathrm{g} \mathrm{L}^{-1}\right)\end{array}$}} & \multirow[b]{2}{*}{ Intercept } & \multirow[b]{2}{*}{ Slope } & \multirow[b]{2}{*}{$\mathrm{s}_{\mathrm{yx}}$} & \multirow{2}{*}{$\begin{array}{l}\text { Error }^{2} \\
(\%)\end{array}$} & \multirow{2}{*}{$\begin{array}{l}\text { Recovery } \\
(\%)\end{array}$} & \multirow{2}{*}{$\begin{array}{l}\text { Repeatability } \\
\left(\mu \mathrm{g} \mathrm{L}^{-1}\right)\end{array}$} & \multicolumn{2}{|c|}{$x=0$} & \multicolumn{2}{|c|}{$\mathrm{x}=\mathrm{MRL}$} \\
\hline & & & & & & & & & & $\begin{array}{c}\mathrm{CC} \alpha \\
\left(\mu \mathrm{g} \mathrm{kg}^{-1}\right)\end{array}$ & $\begin{array}{c}\mathrm{CC} \beta \\
\left(\mu \mathrm{g} \mathrm{kg}^{-1}\right)\end{array}$ & $\begin{array}{c}\mathrm{CC} \alpha \\
\left(\mu \mathrm{g} \mathrm{kg}^{-1}\right)\end{array}$ & $\begin{array}{c}\mathrm{CC} \beta \\
\left(\mu \mathrm{g} \mathrm{kg}^{-1}\right)\end{array}$ \\
\hline \multirow{9}{*}{$\begin{array}{l}\text { Matrix-matched } \\
\text { standards }\end{array}$} & $\mathrm{SZ}$ & 8 & $0-20$ & $6.26 \times 10^{-5}$ & 1 & 0.3736 & 5.94 & 36.73 & 0.57 & 0.81 & 1.55 & 10.78 & 11.51 \\
\hline & $\mathrm{AZ}$ & 10 & $0-20$ & $7.74 \times 10^{-5}$ & 1 & 0.3532 & 8.35 & 61.13 & 1.15 & 0.72 & 1.39 & - & - \\
\hline & $\mathrm{TZ}$ & 6 & $0-5$ & $2.29 \times 10^{-4}$ & 1 & 0.2320 & 9.29 & 57.24 & 1.77 & 0.61 & 1.16 & - & - \\
\hline & \multirow{2}{*}{$\mathrm{ST}$} & 6 & $0-5$ & $-1.79 \times 10^{-5}$ & 1 & 0.2497 & 10.10 & 73.83 & 1.42 & 0.63 & 1.19 & - & - \\
\hline & & 6 & $1-20$ & $3.19 \times 10^{-7}$ & 1 & 0.7277 & - & - & - & - & - & 10.37 & 10.71 \\
\hline & \multirow{2}{*}{$\mathrm{AT}$} & 7 & $0-5$ & $-2.85 \times 10^{-4}$ & 1 & 0.3114 & 10.60 & 83.17 & 1.90 & 0.74 & 1.41 & - & - \\
\hline & & 7 & $1-15$ & $1.16 \times 10^{-7}$ & 1 & 0.4851 & - & - & - & - & - & 11.25 & 12.38 \\
\hline & PT & 9 & $0-15$ & $3.47 \times 10^{-5}$ & 1 & 0.2454 & 12.60 & 64.06 & 1.47 & 0.51 & 0.99 & 10.52 & 11.01 \\
\hline & $\mathrm{TT}$ & 9 & $0-15$ & $1.69 \times 10^{-4}$ & 1 & 0.7211 & 5.40 & 81.43 & 1.74 & 1.05 & 2.04 & 11.46 & 12.83 \\
\hline \multirow{9}{*}{ Standards } & $\mathrm{SZ}$ & 7 & $0-20$ & $-9.09 \times 10^{-6}$ & 1 & 0.4998 & 3.51 & - & 0.37 & 1.15 & 2.21 & 11.08 & 12.08 \\
\hline & \multirow{2}{*}{$\mathrm{AZ}$} & 7 & $0-20$ & $2.14 \times 10^{-6}$ & 1 & 0.4648 & 3.77 & - & 0.16 & 1.07 & 2.21 & - & - \\
\hline & & 7 & $20-80$ & $1.79 \times 10^{-5}$ & 1 & 0.2384 & - & - & - & - & - & 50.51 & 50.99 \\
\hline & \multirow{2}{*}{$\mathrm{TZ}$} & 7 & $0-20$ & $2.41 \times 10^{-5}$ & 1 & 0.3958 & 3.35 & - & 0.11 & 0.91 & 1.75 & - & - \\
\hline & & 7 & $70-130$ & $3.57 \times 10^{-5}$ & 1 & 1.7329 & - & - & - & - & - & 103.7 & 107.2 \\
\hline & ST & 7 & $0-20$ & $4.81 \times 10^{-6}$ & 1 & 0.4125 & 4.20 & - & 0.57 & 0.95 & 1.82 & 10.9 & 11.72 \\
\hline & $\mathrm{AT}$ & 7 & $0-20$ & $6.95 \times 10^{-6}$ & 1 & 0.3901 & 7.29 & - & 0.57 & 0.9 & 1.73 & 10.85 & 11.63 \\
\hline & $\mathrm{PT}$ & 7 & $0-20$ & $8.56 \times 10^{-6}$ & 1 & 0.2322 & 2.42 & - & 0.31 & 0.53 & 1.03 & 10.5 & 10.97 \\
\hline & $\mathrm{TT}$ & 7 & $0-20$ & $3.26 \times 10^{-5}$ & 1 & 0.2596 & 1.28 & - & 0.26 & 0.6 & 1.15 & 10.56 & 11.08 \\
\hline
\end{tabular}




\section{FIGURE CAPTIONS}

Fig. 1 Total ion chromatograms (TICs) from the injection of: (a) a standard of $10 \mu \mathrm{g} \mathrm{L}{ }^{-1}$ of SZ, AZ, PZ (internal standard), TZ, ST, AT, PT and TT, injected in both the injection conditions of experiments 2 and 11 (chromatogram with the largest abundances) in Table 2; (b) the extract of a orange sample fortified with $10 \mu \mathrm{g} \mathrm{L}^{-1}$ of SZ, AZ, TZ, ST, AT, PT and TT and extracted according to the experiment 4 in Table 3 (the extract also contained $10 \mu \mathrm{g} \mathrm{L}^{-1}$ of PZ); and (c) the extract of a orange

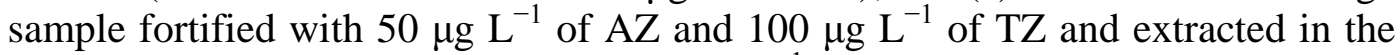
optimal conditions (it also contained $75 \mu \mathrm{g} \mathrm{L} \mathrm{L}^{-1}$ of PZ). Peak labels: 1: SZ; 2: AZ; 3: PZ; 4: TZ; 5: ST; 6: AT; 7: PT; and 8: TT.

Fig. 2 Graphic analysis of the effects of PTV injection factors on the response (standardized loadings of PARAFAC models). Factors: 1, Vent flow; 2, Inlet P; 3, Vent time; 4, Initial temperature; 5, PTV initial time; 6, PTV rate; 7, End temperature and 8, Injection speed. The dash-dotted lines represent the confidence interval of the calculated effects at 95\% confidence level; significant effects (light orange) and non-significant effects (dark blue) .

Fig. 3 Abundances (counts) acquired for AZ for standards (blue continuous line) and orange extracts of experiments in Table 5 (orange dotted line). Diagnostic ions: (a) 173, (b) 200, (c) 202, (d) 215 and (e) 217.

Fig. 4 Loadings of the (a) chromatographic, (b) spectral, and (c) sample modes of the PARAFAC2 model built for AZ in the optimization of the QuEChERS procedure (chromatographic loadings are scaled loadings). First factor is in blue continuous line (blue solid bars in the spectral mode and blue points in the sample mode), second factor is in green dashed line (green dashed bars in the spectral mode and green triangles in the sample mode), and third factor is in red dotted line (red pointed bars in the spectral mode and red squares in the sample mode).

Fig. 5 (a) Loadings of the sample mode of the PARAFAC2 model built for PZ (first factor, blue points; and second factor, green triangles) and (b) standardized loadings of the sample mode calculated for AZ in the optimization of the QuEChERS procedure.

Fig. 6 Graphic analysis of effects of factors of QuEChERS procedure on the response (standardized loadings of PARAFAC2 models). Factors: 1, Vent flow; 2, Inlet P; 3, Vent time; 4, Initial temperature; 5, PTV initial time; and 6, PTV rate. For each factor the levels chosen are in solid bars.

Fig. 7 Loadings of the (a) chromatographic, (b) spectral, and (c) sample modes and (d) standardized loadings of the sample mode of the PARAFAC2 model built for AZ in the quantitative and qualitative determination of triazines in oranges (chromatographic loadings are scaled loadings). First factor is in blue continuous line (blue solid bars in the spectral mode and blue points in the sample mode), second factor is in green dashed line (green dashed bars in the spectral mode and 
green triangles in the sample mode), third factor is in red dotted line (red pointed bars in the spectral mode and red squares in the sample mode), and fourth factor is in cyan dash-dot-dot line (cyan transparent bars in the spectral mode and cyan stars in the sample mode).

Fig. 8 Joint confidence ellipses, at 95\% confidence level, for the slope and the intercept of the regression models "calculated concentration $v$ s. true concentration" in Table 8 for (a) matrix-matched standards and (b) standards. SZ: solid blue line; AZ: red triangles; TZ: green circles; ST: black dotted line; AT: magenta dash-dot line; PT: cyan squares; and TT: yellow dashed line. 

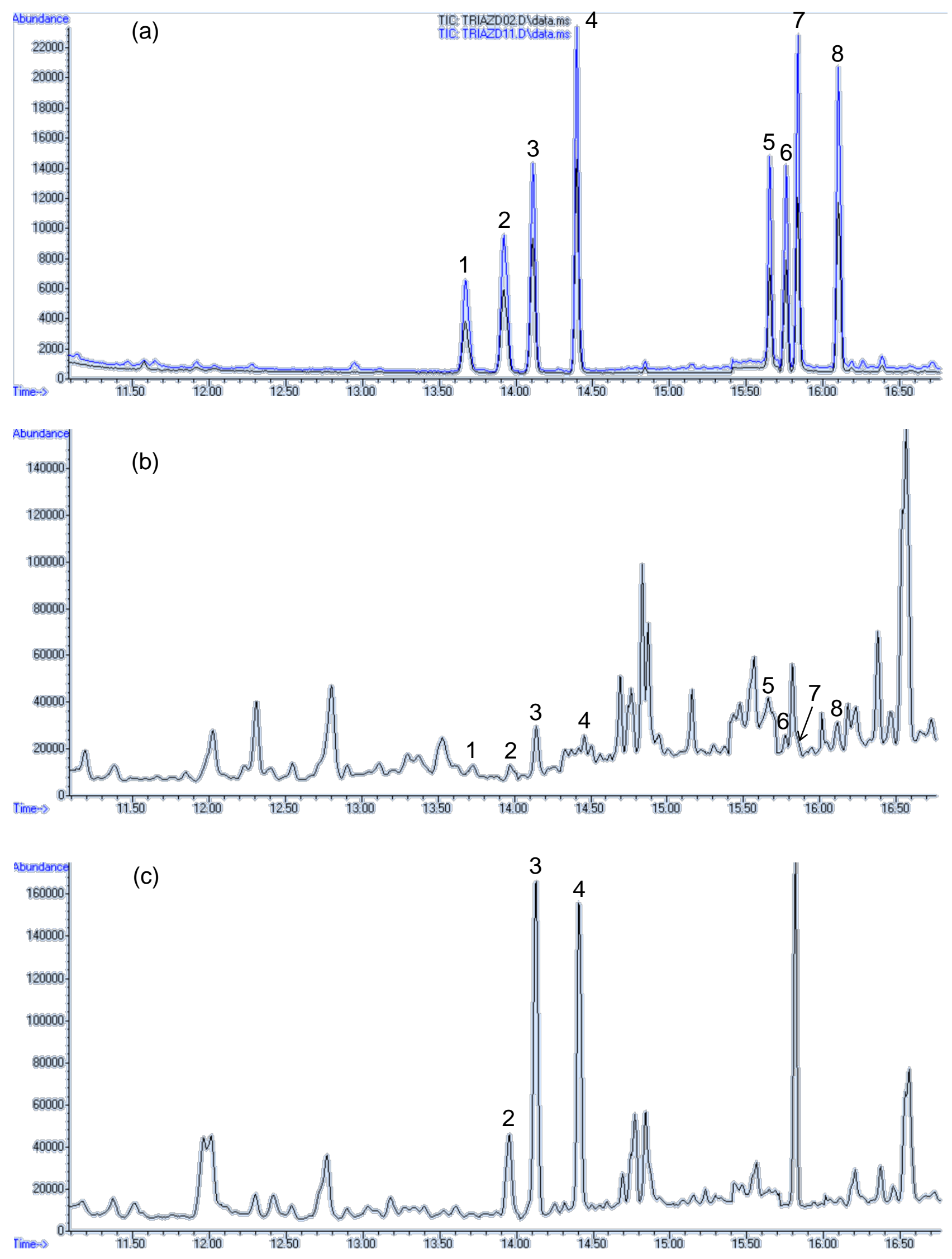

FIGURE 1 


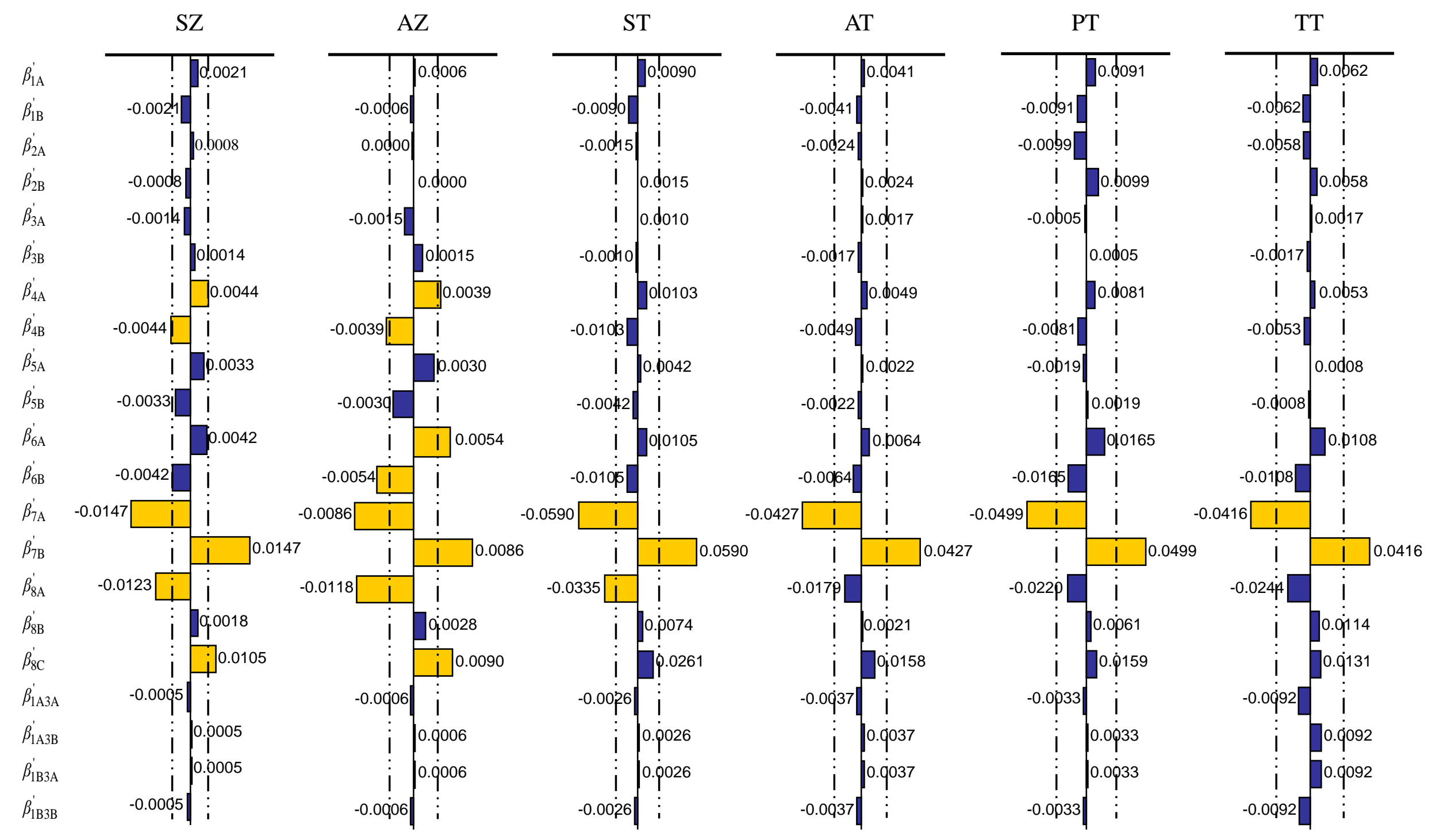



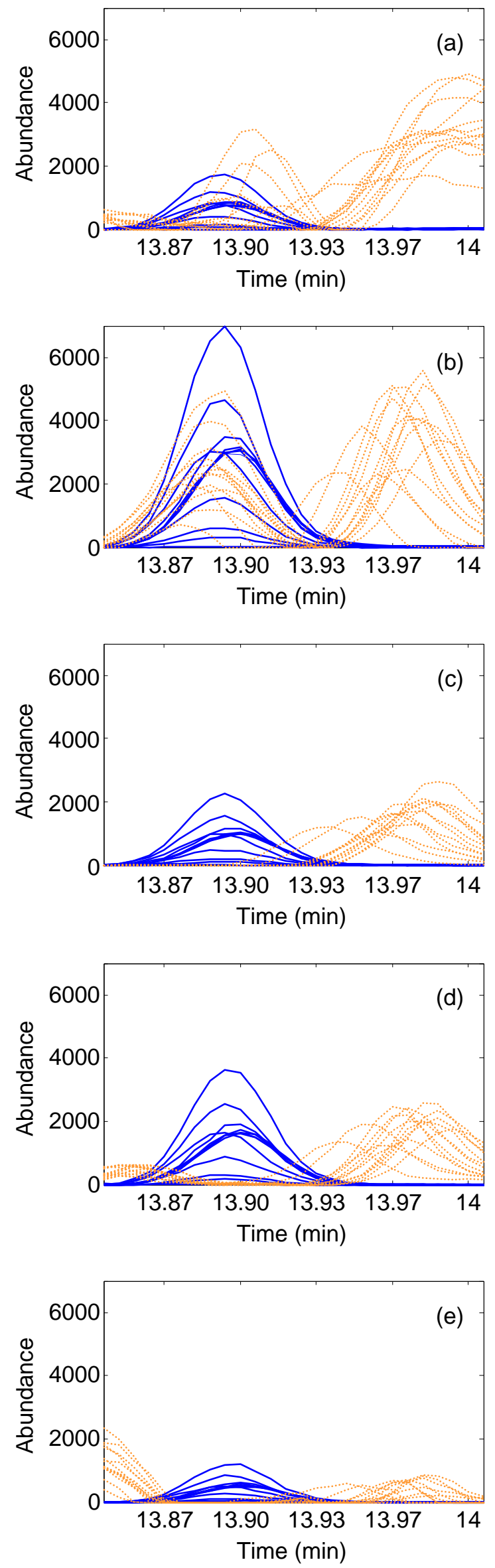

FIGURE 3 

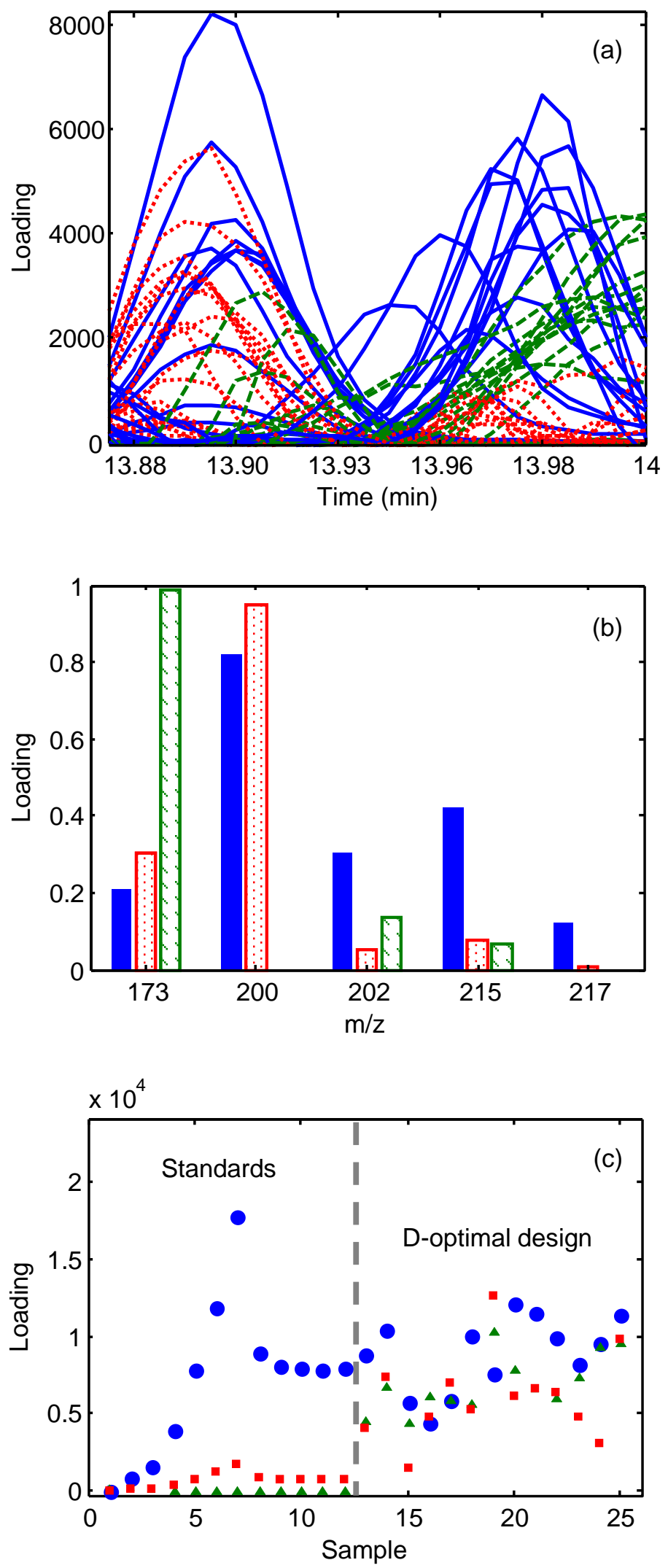

FIGURE 4 

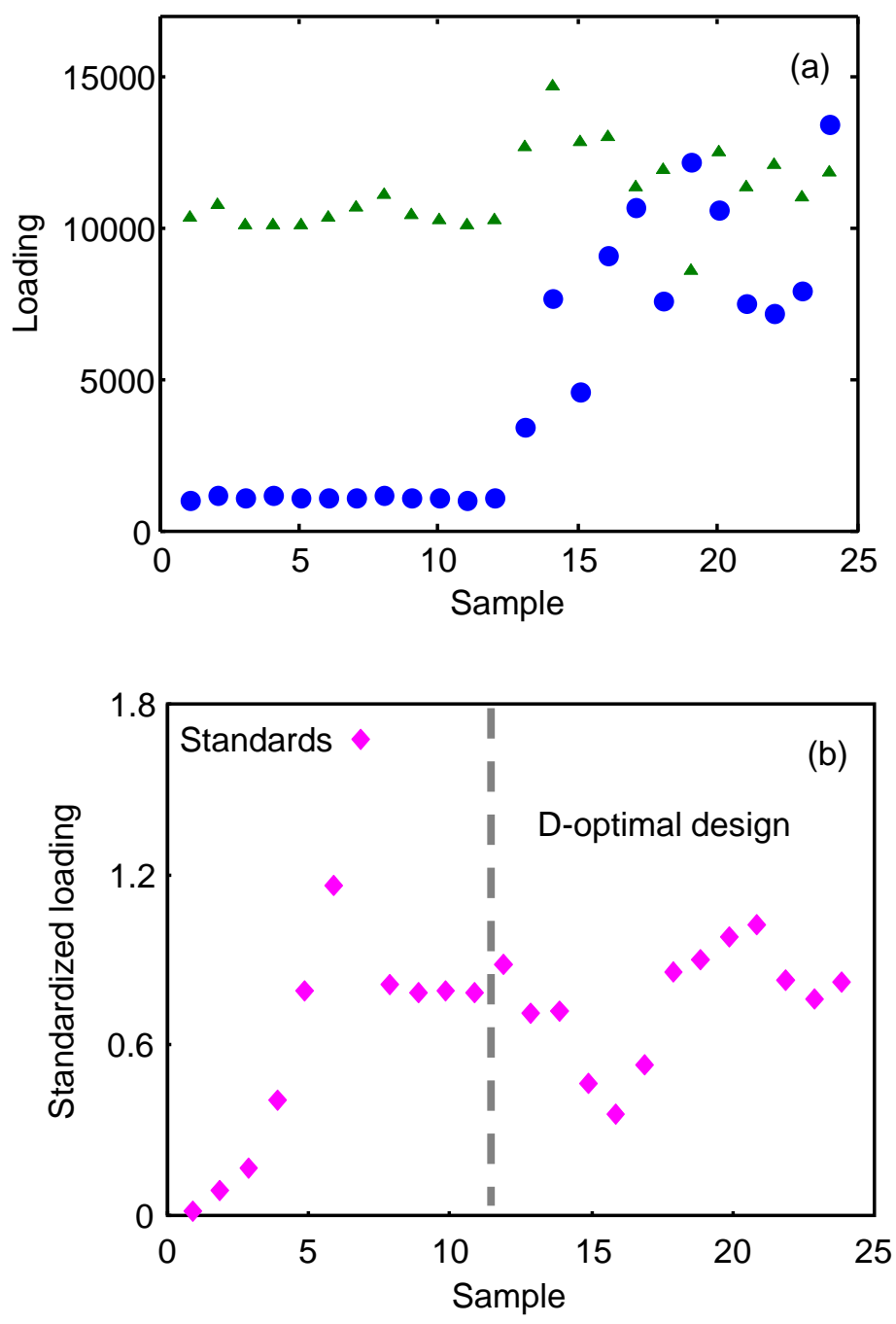

FIGURE 5 

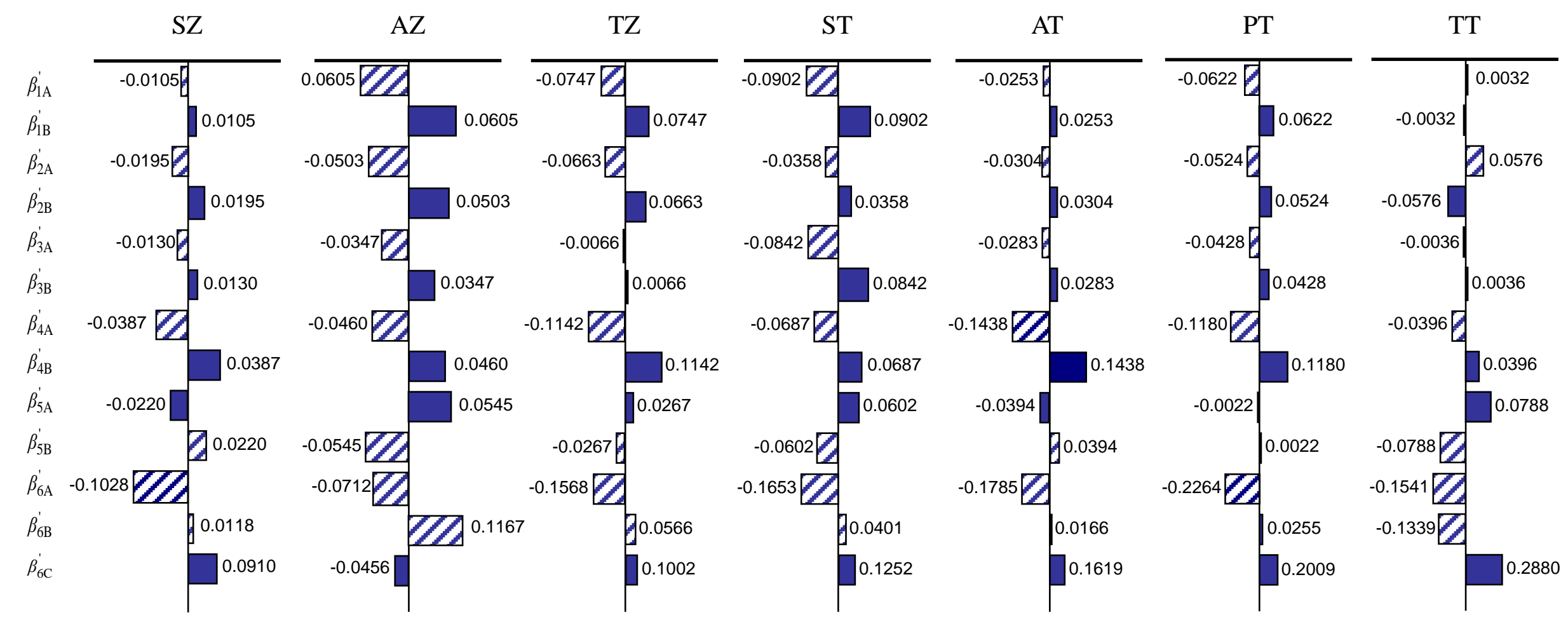

FIGURE 6 

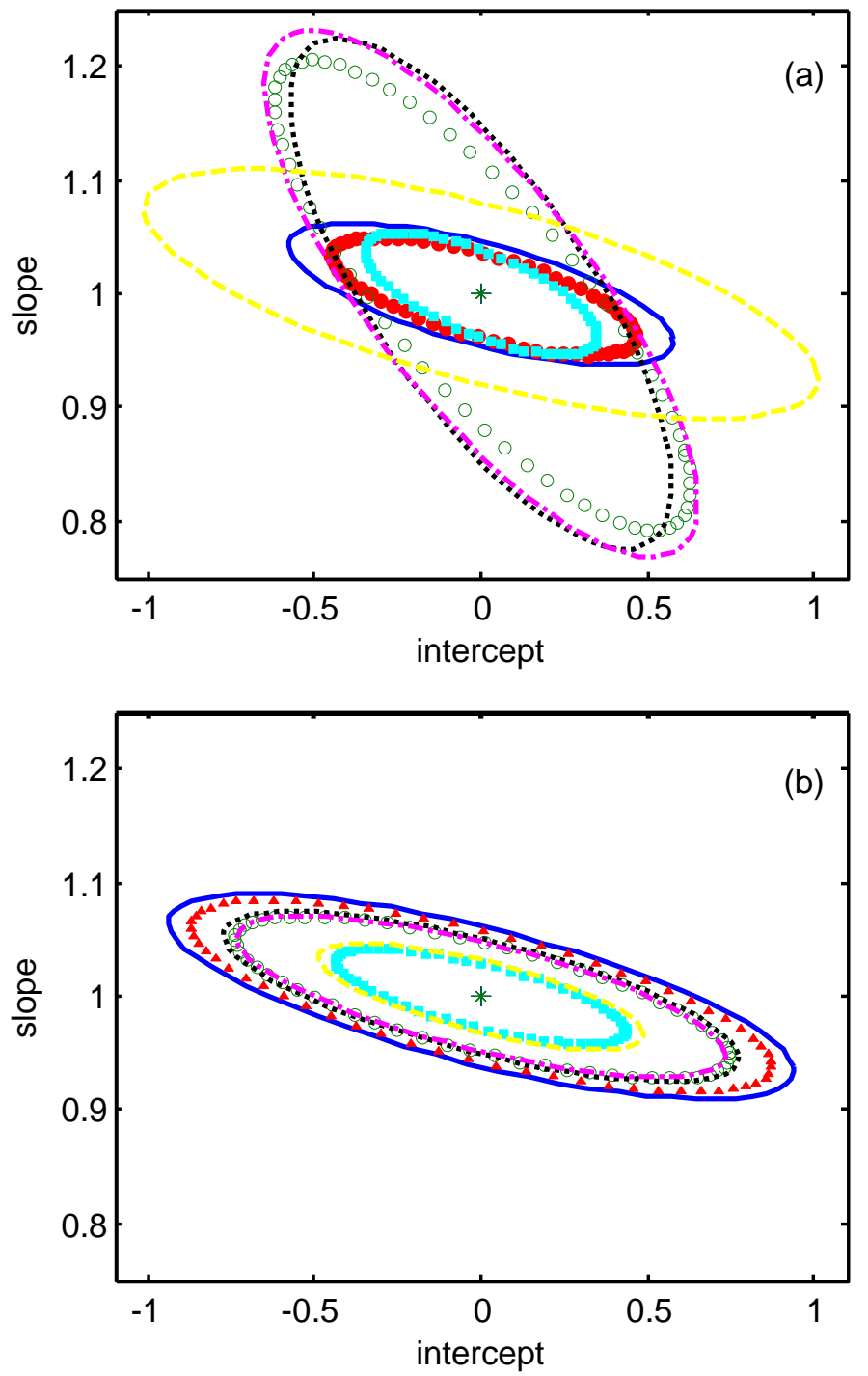

FIGURE 8 